\title{
STING activation reprograms tumor vasculatures and synergizes with VEGFR2 blockade
}

\author{
Hannah Yang, ${ }^{1,2}$ Won Suk Lee, ${ }^{1,2}$ So Jung Kong, ${ }^{1,2}$ Chang Gon Kim, ${ }^{3}$ Joo Hoon Kim,,${ }^{1,2}$ Sei Kyung Chang, ${ }^{4}$ Sewha Kim, ${ }^{5}$ Gwangil Kim, \\ Hong Jae Chon, ${ }^{1,2}$ and Chan $\mathrm{Kim}^{1,2}$ \\ 1Medical Oncology, CHA Bundang Medical Center, CHA University, Seongnam, South Korea. ²aboratory of Translational Immuno-Oncology, CHA University, Seongnam, South Korea. ${ }^{3}$ Graduate School of \\ Medical Science and Engineering, Korea Advanced Institute of Science and Technology (KAIST), Daejeon, South Korea. ${ }^{4}$ Department of Radiation Oncology and ${ }^{5}$ Department of Pathology, CHA Bundang \\ Medical Center, CHA University, Seongnam, South Korea.
}

\begin{abstract}
The stimulator of IFN genes (STING) signaling pathway is a critical link between innate and adaptive immunity and induces antitumor immune responses. STING is expressed in vasculatures, but its role in tumor angiogenesis has not been elucidated. Here, we investigated STING-induced tumor vascular remodeling and the potential of STING-based combination immunotherapy. Endothelial STINC expression was correlated with enhanced T cell infiltration and prolonged survival in human colon and breast cancer. Intratumoral STING activation with STING agonists (cCAMP or RR-CDA) normalized tumor vasculatures in implanted and spontaneous cancers, but not in STING-deficient mice. These were mediated by upregulation of type I/II IFN genes and vascular stabilizing genes (e.g., Angpt1, Pdgfrb, and Col4a). STING in nonhematopoietic cells is as important as STING in hematopoietic cells for inducing a maximal therapeutic efficacy of exogenous STING agonists. Vascular normalizing effects of STING agonists were dependent on type I IFN signaling and CD8+ T cells. Notably, STING-based immunotherapy was maximally effective when combined with VEGFR2 blockade and/or immune-checkpoint blockade ( $\alpha$ PD-1 or $\alpha$ CTLA-4), leading to complete regression of immunotherapy-resistant tumors. Our data show that intratumoral STING activation can normalize tumor vasculature and the tumor microenvironment, providing a rationale for combining STINGbased immunotherapy and antiangiogenic therapy.
\end{abstract}

\section{Introduction}

The stimulator of IFN genes (STING) signaling pathway is the critical innate immune sensor for tumor detection (1-4). Upon activation in antigen-presenting cells within a tumor microenvironment (TME), the STING pathway drives robust production of type I IFNs and enhances $\mathrm{CD}^{+} \mathrm{T}$ cell crosspriming by tumor antigens, ultimately inducing adaptive anticancer immune responses (4-6). Based on these actions, various STING agonists have been developed for cancer therapy; these show promising antitumor activities as monotherapy or combined with other therapeutics (6-11). However, while the STING pathway's role in dendritic cells is largely delineated, its contribution in other cellular components of the TME is not yet well elucidated $(4,5,12)$.

The inner lining of tumor vasculature comprises tumor endothelial cells that serve as a biologic interface between tumor and systemic immunity (13-15). The dynamic interplay between endothelium and immune cells is important for immune cell trafficking across the endothelial barrier and determines the quality and amplitude of anticancer immune responses (16-19). Unfortunately, most tumor blood vessels are structurally malformed and func-

Authorship note: HJC and CK contributed equally to this work. Conflict of interest: The authors have declared that no conflict of interest exists. Copyright: () 2019, American Society for Clinical Investigation.

Submitted: October 8, 2018; Accepted: July 18, 2019; Published: September 9, 2019. Reference information: J Clin Invest. 2019;129(10):4350-4364. https://doi.org/10.1172/JCl125413. tionally anergic, thus limiting $\mathrm{T}$ lymphocyte survival and effector functions and suppressing antitumor immune responses in the $\operatorname{TME}(15,16,20)$. Alleviating these vascular abnormalities - a process known as vascular normalization - may relieve intratumoral immunosuppression and eventually enhance cancer immunotherapy efficacy $(14,15,21,22)$.

Multiple lines of evidence suggest that STING signaling may be important in vascular biology. First, the STING protein is expressed in endothelial cells of both normal and tumor tissue $(6,12,23)$. Particularly strong STING expression is observed in high-endothelial venule, which is a specialized endothelium for lymphocyte migration in lymphoid organs, suggesting the potential involvement of STING signaling in endothelial-lymphocyte interaction (24). Second, in various murine and human tumors, STING activation prompts endothelial cells to produce type I IFNs, especially IFN- $\beta$ $(6,23)$. Notably, endothelial cell-derived type I IFNs initiate antitumor responses even before dendritic cells and $\mathrm{CD} 8^{+} \mathrm{T}$ cells infiltrate the TME and determine the overall magnitude of local and systemic immunity (23). Third, excessive STING activation is associated with aberrant vascular activation. Patients with a rare genetic syndrome, now termed STING-associated vasculopathy with onset in infancy (SAVI), commonly exhibit mutations in the STING-encoding gene TMEM173, which lead to constitutive activation of IFN- $\beta$ and IFN-response genes, resulting in fatal vasculitis $(25,26)$.

The presently available data suggest that endothelial cells may be another important target of STING agonists and that STING-induced activation of endothelial cells could promote 

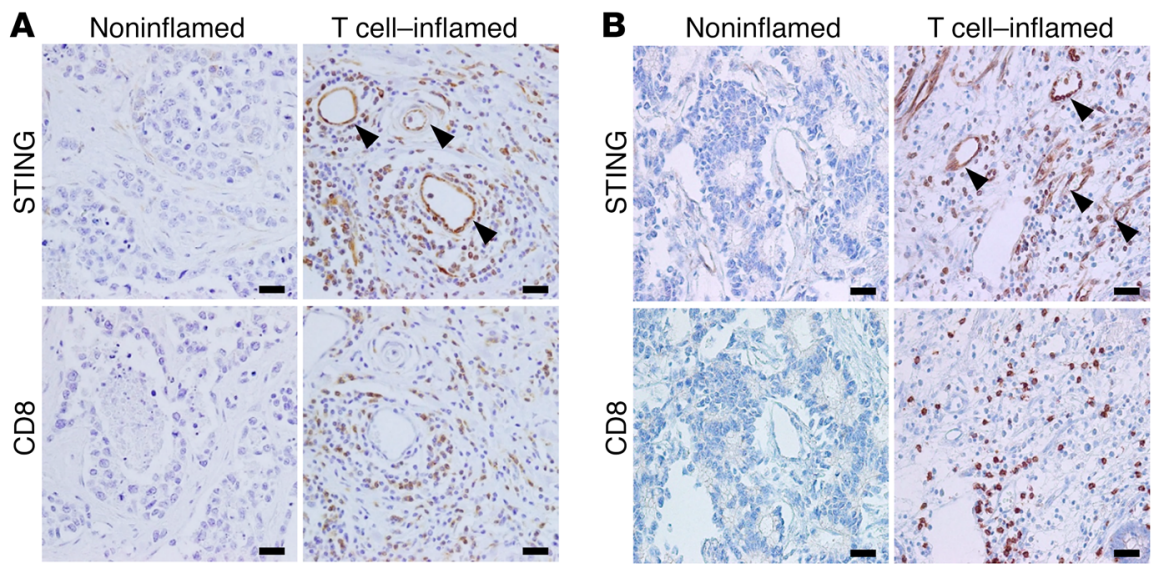

Figure 1. Endothelial STING expression correlates with intratumoral $\mathrm{CD8}^{+} \mathrm{T}$ cell infiltration and favorable prognosis in human cancers. Clinical implications of endothelial STING expression (Endo STING) in breast cancer $(n=173)$ and colorectal cancer $(n=160)$. (A and B) Representative images of STING and CD8 expression in human breast cancer (A) and colorectal cancer (B). (C and D) Correlation between endothelial STING expression and intratumoral $\mathrm{CD} 8^{+}$cells in breast cancer (C) and colorectal cancer (D). $R$ and $P$ values by Pearson's correlation test. (E and F) Kaplan-Meier survival curves of breast cancer patients $(\mathbf{E})$ and colorectal cancer patients (F) using endothelial STING expression. $P$ values by the log-rank test.

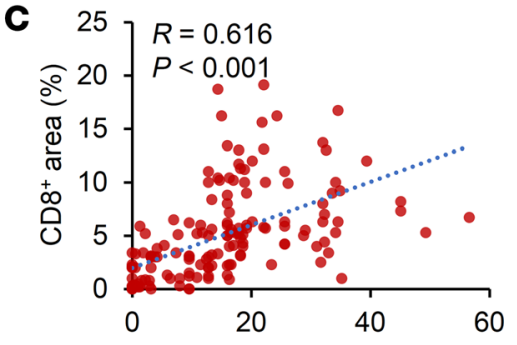

Endothelial STING expression (\%)

$\mathbf{E}$

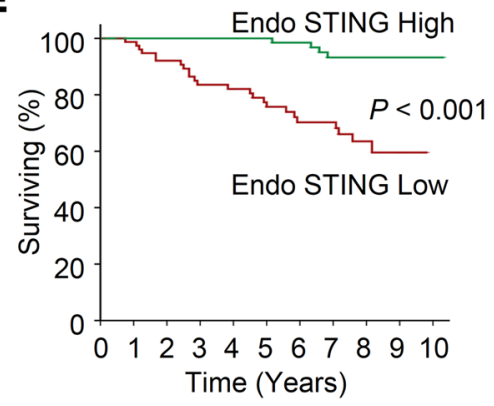

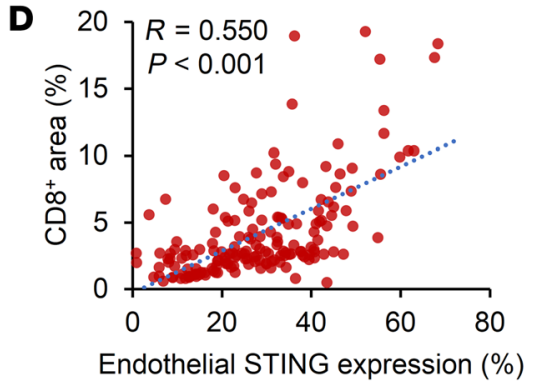

$\mathbf{F}$

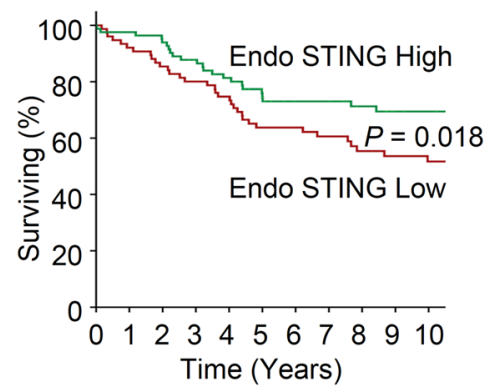

phenotypic and functional changes of tumor blood vessels. In the present study, we demonstrated the normalization of tumor vasculature upon STING activation and the therapeutic relevance of STING-targeted therapy in various tumor models, particularly when combined with an antiangiogenic agent.

\section{Results}

Endothelial STING expression correlates with intratumoral $C D 8^{+} T$ cell infiltration and favorable prognosis in human cancers. To explore the clinical relevance of endothelial STING expression in human malignancies, we assessed the STING expression pattern in tumor tissues from 173 breast and 160 colorectal cancer patients. We detected distinct STING expression in tumor endothelial cells and immune cells (Figure 1, A and B, and Supplemental Table 1; supplemental material available online with this article; https:// doi.org/10.1172/JCI125413DS1). Intriguingly, CD8 ${ }^{+} \mathrm{T}$ cell infiltration was increased near STING-expressing tumor vessels and was significantly correlated with endothelial STING expression levels (Figure 1, C and D). Moreover, compared with those with low STING expression, patients with high endothelial STING expression had better overall survival after cancer diagnosis (Figure 1,
E and F, and Supplemental Figure 1, A and B). High endothelial STING expression was also correlated with decreased prevalence of lymphovascular invasion within tumor tissues (Supplemental Table 2). This prognostic significance remained true even in multivariate Cox regression analysis using various clinical and molecular characteristics (Supplemental Table 3).

To gain deeper understanding of the role of endothelial STING in tumor growth, we utilized several mouse models. STING was expressed in $\mathrm{CD} 1^{+}$tumor endothelial as well as in hematopoietic cells, such as dendritic cells and macrophages (Supplemental Figure 2A); however, the level of STING expression in endothelial cells varied among different tumor models, with the CT26 colon cancer and Lewis lung carcinoma (LLC) models showing stronger endothelial STING expression compared with the MMTV-PyMT breast cancer model (Supplemental Figure 2, B and C). Intriguingly, consistent with the findings from human cancers, levels of endothelial STING expression were also significantly correlated with intratumoral $\mathrm{CD}^{+} \mathrm{T}$ cells in mouse tumor models $(P=0.001$, $\mathrm{R}=0.601$ ) (Supplemental Figure 2D). Moreover, CT26, the tumor with modest STING expression and the most abundant intratumoral $\mathrm{CD}^{+} \mathrm{T}$ cells, showed the best response to treatment with 
A

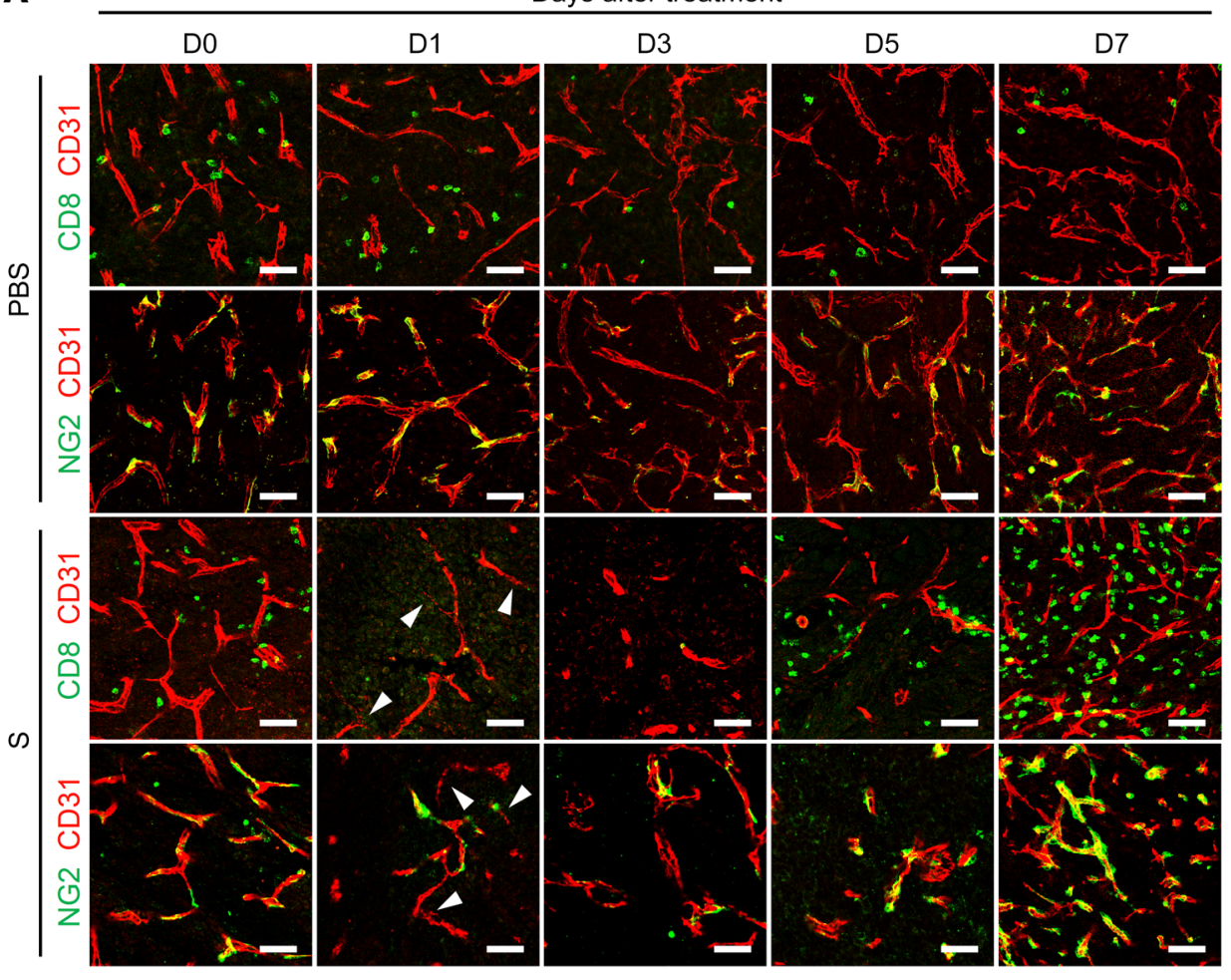

B

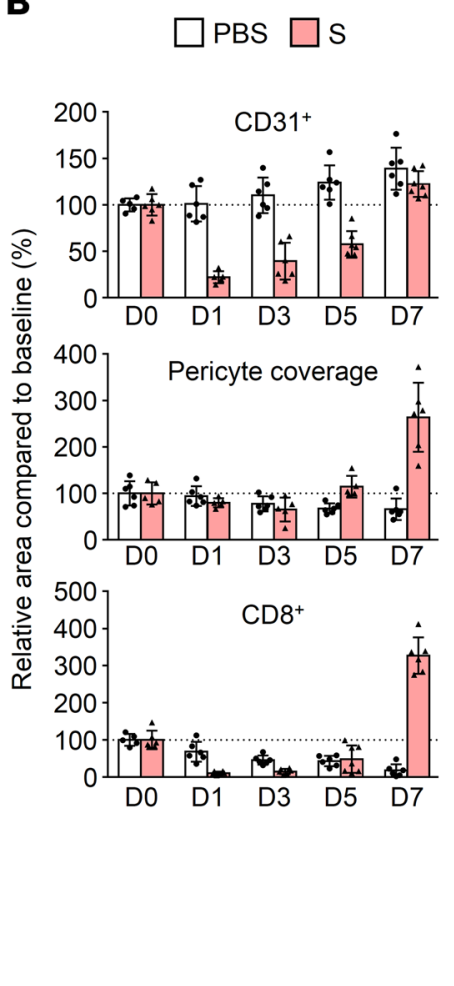

Figure 2. Intratumoral injection of STING agonist induces dramatic changes in TME. LLC tumor cells were implanted subcutaneously into mice and treated with intratumoral injections of PBS or STING agonists (S). (A) Serial images of LLC tumors after a single injection of PBS or STING agonist (RR-CDA, $25 \mu \mathrm{g})$. Arrowheads indicate disrupted tumor vessels. (B) Temporal changes in CD31+ blood vessels, NG2 ${ }^{+}$pericyte coverage, and CD8 ${ }^{+} \mathrm{T}$ cells after a single injection of PBS or STING agonist treatment. Pooled data from 2 independent experiments with $n=5$ to 6 per group. Scale bars: $50 \mu \mathrm{m}$.

STING agonists, while MMTV-PyMT, the tumor with the weakest STING expression, did not respond as well to treatment with a STING agonist alone (Supplemental Figure 2E). Therefore, the levels of baseline STING expression and intratumoral $\mathrm{CD}^{+} \mathrm{T}$ cells may be predictors of therapeutic response to STING agonist monotherapy. Collectively, these findings suggest that endothelial STING signaling is associated with the prognosis of cancer patients and may play an important role during $\mathrm{CD}^{+} \mathrm{T}$ cell-mediated anticancer immunity.

STING agonists promote $C D 8^{+} T$ cell responses and tumor vascular normalization. We next examined the temporal changes of tumor vasculatures and $\mathrm{CD} 8^{+} \mathrm{T}$ cells after intratumoral injection of STING agonist into LLC tumors (Figure 2, A and B). A single injection of RR-CDA (also called MIW815 or ADU-S100) led to dramatic changes in TME compared with that seen in PBS-injected control tumors. Whereas consistent angiogenesis was observed in control tumors, RR-CDA-treated tumors showed an abrupt decrease in CD31+ tumor vessels at 1 day after RR-CDA treatment, followed by a gradual recovery. In addition, control tumors showed a slow decline in $\mathrm{NG}^{+}$pericyte coverage and a gradual decrease in $\mathrm{CD}^{+}{ }^{+} \mathrm{T}$ cells, while RR-CDA-treated tumors showed increased pericyte coverage and a dramatic influx of $\mathrm{CD}^{+} \mathrm{T}$ cells at 7 days after RR-CDA treatment. Of note, increased pericyte coverage, one of the hallmarks of tumor vessel normalization, coincided with the time of peak $\mathrm{CD} 8^{+} \mathrm{T}$ cell infiltration into the TME.
To further dissect the effects of intratumoral STING activation on TME, LLC tumor-bearing mice were treated with repeated injections of the STING agonists cGAMP or RR-CDA. After 3 consecutive intratumoral cGAMP injections, tumor growth was reduced by $46 \%$ compared with that in controls (Figure $3 \mathrm{~A}$ ). TME analyses revealed that cGAMP treatment led to a 6.4-fold increase in intratumoral $\mathrm{CD}^{+} \mathrm{T}$ cells, $40 \%$ reduction in $\mathrm{CD} 31^{+}$ blood vessel density, a 1.7 -fold increase in $\mathrm{NG} 2^{+}$pericyte coverage, and a 1.5 -fold increase in COL $4^{+}$basement membrane coverage. Additionally, intratumoral hypoxia was alleviated by $46 \%$ in cGAMP-treated tumors compared with controls (Figure 3, B and $\mathrm{C}$ ). Two injections of RR-CDA induced similar changes in the TME of LLC tumors: $41 \%$ delayed tumor growth, a 16.5 -fold increase in intratumoral CD8 ${ }^{+} \mathrm{T}$ cells, a $51 \%$ decrease in CD $31^{+}$ blood vascular density, a 4.4 -fold increase in $\mathrm{NG}^{+}$pericyte coverage, and a 3.1-fold increase in COL $4^{+}$basement membrane coverage compared with controls. Moreover, glucose transporter 1 (GLUT1) levels were analyzed as hypoxia markers, as previously described $(21,27)$, revealing a $46 \%$ decrease in intratumoral hypoxia after RR-CDA injection compared with that in controls (Figure 3, D-F).

In order to define the dose-ranging effect of STING agonist, we intratumorally injected 1 to $100 \mu \mathrm{g}$ of RR-CDA into LLC tumors. RR-CDA effectively suppressed tumor growth even with a dose of $1 \mu \mathrm{g}$ (Supplemental Figure 3, A and B), but its antiangiogenic and vascular normalizing effect was seen at a dose of $5 \mu \mathrm{g}$ or more 
A

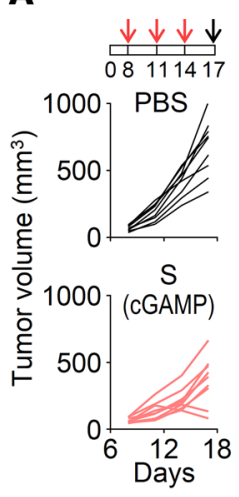

B

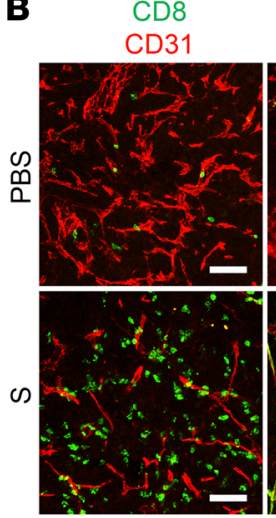

$\mathbf{E}$

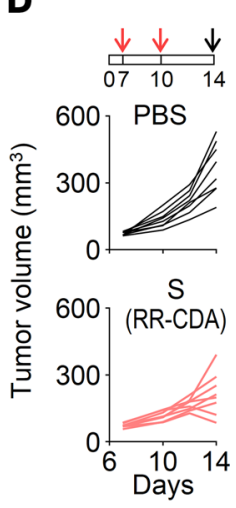

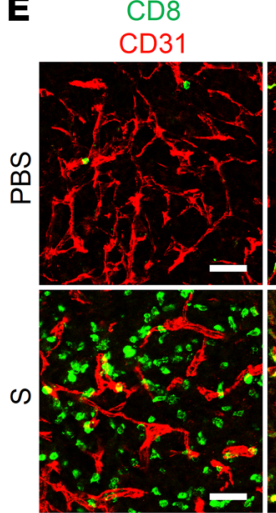
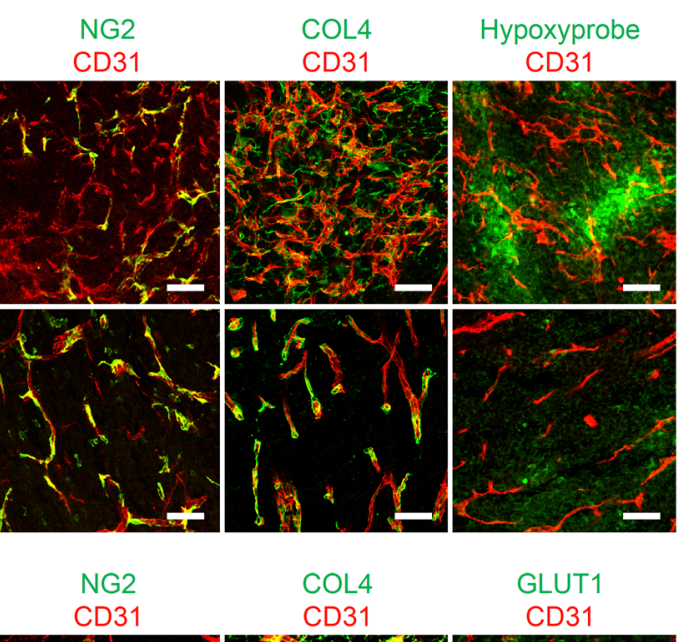

NG2
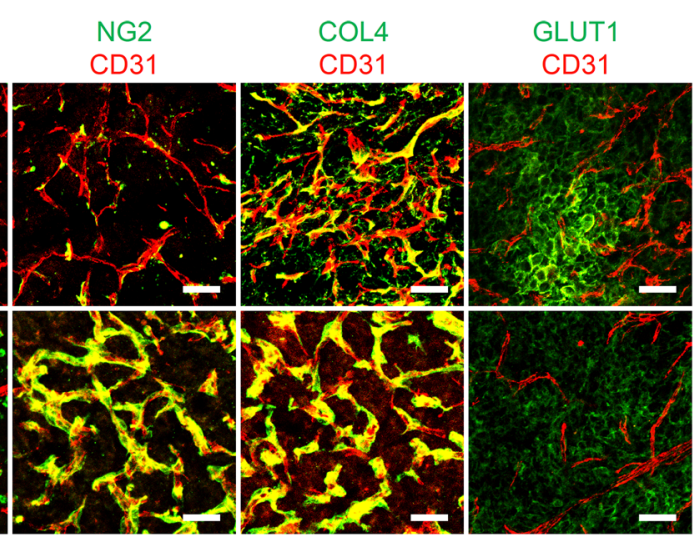

Hypoxyprobe

GLUT1 CD31
C

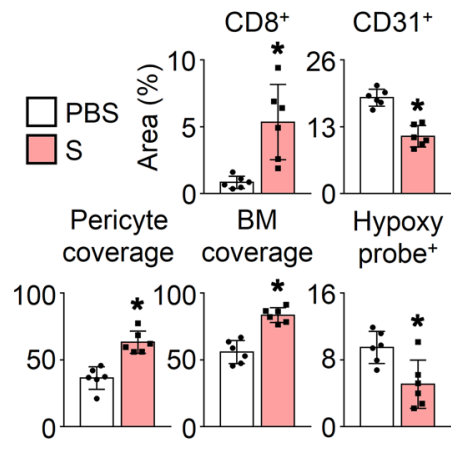

$\mathbf{F}$

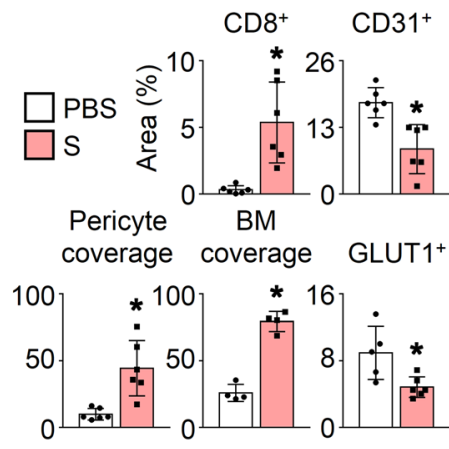

Figure 3. STING agonists promote CD8+ T cell responses and tumor vascular normalization. LLC tumor cells were implanted subcutaneously into mice and treated with intratumoral injections of PBS or STING agonists (S). Red arrows indicate treatment, and black arrows indicate sacrifice. (A) Comparison of LLC tumor growth in mice treated with PBS or STING agonist (cCAMP, $10 \mu \mathrm{g})$. (B and C) Representative images (B) and comparisons (C) of CD8 ${ }^{+}$T cells, CD31+ blood vessels, NG2+ pericyte coverage, COL4+ basement membrane (BM) coverage, and hypoxic area. (D) Comparison of LLC tumor growth in mice treated with PBS or STING agonist (RR-CDA, $25 \mu \mathrm{g}$ ). (E and F) Representative images (E) and comparisons (F) of CD8 ${ }^{+}$T cells, CD31+ blood vessels, NG2+ pericyte coverage, COL4+ basement membrane coverage, and GLUT1 ${ }^{+}$hypoxic area. Pooled data from 2 independent experiments with $n=8$ to 9 per group. Values are shown as mean \pm SD. ${ }^{*} P<0.05$ versus PBS. Two-tailed Student's $t$ test. Scale bars: $50 \mu \mathrm{m}$.

(Supplemental Figure 3, C and D). When the dose of intratumoral RR-CDA was increased up to $100 \mu \mathrm{g}$, all tumors had completely regressed. Overall, these findings indicate that STING activation can augment intratumoral $\mathrm{CD}^{+} \mathrm{T}$ cell infiltration, normalize tumor vessels, and alleviate hypoxia within the tumor.

STING signaling pathway regulates tumor vascular and immune microenvironment. To examine how STING signaling is involved in tumor vascular normalization, we investigated how STING agonist affected the TME in STING-deficient (STING $\left.{ }^{g t / g t}, \mathrm{KO}\right)$ and WT mice (Figure 4). In WT mice, intratumoral administration of cGAMP suppressed LLC tumor growth by $47 \%$, reduced tumor vessel density by $48 \%$ and the number of vascular sprouts by $55 \%$, and increased pericyte coverage by 1.7 -fold. However, no changes in tumor growth or tumor vessels were observed in $\mathrm{KO}$ mice, indicating that the cGAMP-induced antiangiogenic effects were STING dependent. Moreover, in the absence of cGAMP treatment, tumors of KO mice showed 1.4-fold higher blood vessel density, 1.5-fold increased number of vascular sprouts, and $47 \%$ decreased pericyte coverage compared with tumors of WT mice (Figure 4, A-C). Collectively, these findings suggest that STING signaling acts as a suppressor of sprouting tumor angiogenesis and an inducer of tumor vessel maturation.
To elucidate the transcriptional changes upon STING activation, we used the NanoString PanCancer panel to compare 750 immune microenvironment-related genes in cGAMP-treated tumors in WT mice and tumors in KO mice. The results revealed dramatic differences between the STING-activated and STING-deficient TMEs (Figure 5A). At first, intratumoral STING activation strongly induced both type I IFNs and type II IFNs within TME (Figure 5B). Next, vascular stabilizing genes (e.g., Cdh5, Angpt1, Pdgfrb, Mcam, and Col4a) were also increased after STING activation, but were decreased or unchanged in $\mathrm{KO}$ mice (Figure $5 \mathrm{C})$. On the other hand, vascular destabilizing genes did not differ significantly between groups (Figure 5D). We also compared various adhesion molecules that are critical in endothelial-lymphocyte interactions and lymphocyte transmigration and found that STING activation significantly upregulated adhesion molecules, including Icam, Vcam, and Sell (Figure 5E). The full list of other gene changes is provided in Supplemental Table 4 and Supplemental Figure 3, E and F.

Since STING signaling is critical for myeloid cell activation (8), we also analyzed genes involved in macrophage polarization (Supplemental Figure 4, A and B). STING activation was associated with marked increases in genes specific for M1-like 
A
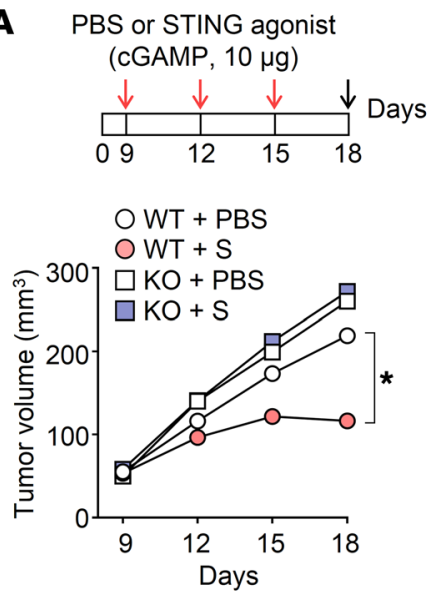

B

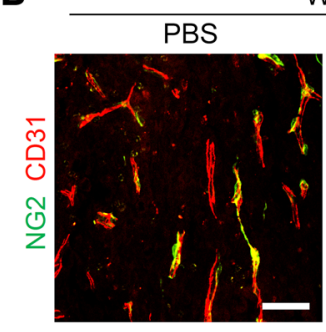

WT

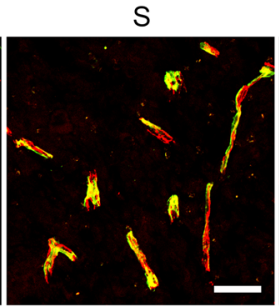

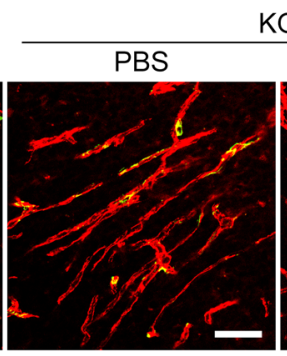

KO

C

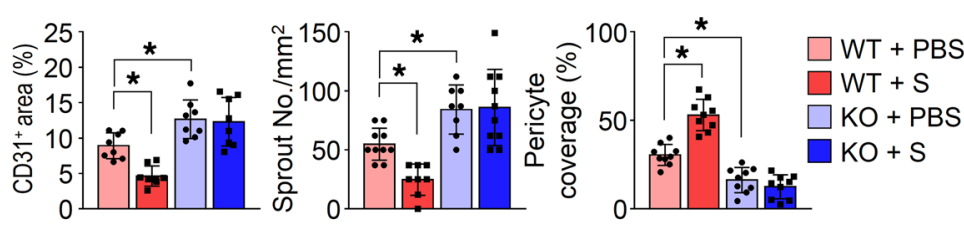

Figure 4. STING signaling pathway regulates tumor angiogenesis. LLC tumor cells were implanted subcutaneously into WT or STING-deficient mice (STING $\left.{ }^{g t / g t}, \mathrm{KO}\right)$ and treated with intratumoral injections of PBS or STING agonist (S). (A) Comparison of tumor growth in mice treated with PBS or STING agonist. Red arrows indicate treatment, and black arrow indicates sacrifice. (B and C) Representative images (B) and comparisons (C) of CD31+ blood vessels, tumor vessel sprouts, and NG2+ pericyte coverage. Pooled data from 2 experiments with $n=8$ per group. Values are shown as mean \pm SD. ${ }^{*} P<0.05$ versus PBS. ANOVA with Tukey's post hoc test (A and $\mathbf{C})$. Scale bars: $50 \mu \mathrm{m}$.

macrophages, while genes for M2-like macrophages were not significantly altered. This was confirmed by treating tumors with cGAMP or RR-CDA, and we found an increase in NOS2 ${ }^{+}$M1-like macrophages, while CD206 ${ }^{+}$M2-like macrophages were not significantly altered compared with control (Supplemental Figure 4, C-E). Consistently, flow cytometric analysis also revealed an accumulation of M1-like macrophages, but no changes in M2-like macrophages, yielding an increasing trend in the M1/M2 ratio (Supplemental Figure 4F). We then examined the role of macrophages after intratumoral STING agonist treatment by selectively depleting macrophages with clodronate liposome (Supplemental Figure 4G) (28). Intriguingly, the antitumor efficacy of RR-CDA did not change significantly even after macrophage depletion (Supplemental Figure 4, H and I). Therefore, although intratumoral STING agonist treatment stimulates the accumulation of M1-like macrophages within the TME, macrophages seem to be dispensable for the overall antitumor effect of STING agonists.

Finally, we found that intratumoral STING activation triggered an increase in inhibitory (e.g., Pd-1, Pd-l1, Ctla-4, Lag-3, and Tim-3) and agonistic (e.g., Icos, Ox4O, Gitr, Hvem, and $\mathrm{Cd27}$ ) immune checkpoint genes (Figure $5 \mathrm{~F}$ ). We also confirmed increases in $\mathrm{PD}-1^{+} \mathrm{CD} 8^{+} \mathrm{T}$ cells, CTLA $-4^{+} \mathrm{CD} 8^{+} \mathrm{T}$ cells, TIM- $3^{+} \mathrm{CD} 8^{+} \mathrm{T}$ cells, and $\mathrm{PD}-\mathrm{L} 1^{+} \mathrm{CD} 45^{-}$cells in TME of STING-treated tumors (Figure $5 \mathrm{G}$ ). Collectively, our findings indicate that activation of STING signaling negatively regulates tumor angiogenesis in the TME and upregulates genes involved in vascular normalization, endothelial-lymphocyte interaction, and immune checkpoints.

STING in nonhematopoietic cells is as important as STING in hematopoietic cells during therapy with exogenous STING agonist. Although the roles of STING signaling are mostly well delineated in hematopoietic immune cells, its role in nonhematopoietic stromal cells is not so well defined, even though these cells also express STING. To investigate the roles of hematopoietic-derived cells or nonhematopoietic stromal cells, such as endothelial cells, in the therapeutic efficacy of STING agonists, we generated chi- meric mice by transferring WT or KO bone marrow into lethally irradiated WT or KO mice into which we subcutaneously implanted LLC tumor cells (Figure 6A). When the tumors were intratumorally treated with RR-CDA, tumor growths were significantly suppressed in WT $\rightarrow$ WT (bone marrow donor, WT; recipient, WT) mice and partially suppressed in $\mathrm{KO} \rightarrow \mathrm{WT}$ or $\mathrm{WT} \rightarrow \mathrm{KO}$ mice, but not suppressed in $\mathrm{KO} \rightarrow \mathrm{KO}$ mice (Figure 6, B and $\mathrm{C}$ ), indicating that both hematopoietic and nonhematopoietic STING is important for the anticancer effects of STING agonist treatment. Intriguingly, $\mathrm{KO} \rightarrow$ WT mice showed decreased tumor angiogenesis and increased pericyte coverage, with less intratumoral CD $8^{+} \mathrm{T}$ cells. On the other hand, WT $\rightarrow$ KO mice showed more intratumoral $\mathrm{CD}^{+} \mathrm{T}$ cells, with less pronounced suppression of tumor angiogenesis and decrease in pericyte coverage (Figure 6, D and E). Therefore, it seems that STING in nonhematopoietic cells is more important in the regulation of tumor vessels, whereas STING in hematopoietic cells is more important in determining the magnitude of anticancer immune response by $\mathrm{CD}^{+} \mathrm{T}$ cells within the tumor. Taken together, these results suggest that STING in nonhematopoietic cells is as important as STING in hematopoietic cells for inducing a maximal therapeutic efficacy of exogenous STING agonist treatment.

$T M E$ regulation by STING activation is dependent on type I IFN signaling and $C D 8^{+} T$ cells. To determine which immune system elements were responsible for STING-induced TME remodeling, we treated tumors with neutralizing antibodies against IFNAR or CD8. IFNAR depletion completely negated and CD8 depletion partially ( $40 \%)$ abrogated the antitumor efficacy of STING agonist treatment (Figure 7, A and B, and Supplemental Figure 5, A and B). Notably, blockade of type I IFN signaling or depletion of $\mathrm{CD}^{+} \mathrm{T}$ cells also abrogated STING agonists' antiangiogenic and vascular normalizing effects (Figure 7, C and D). This also nullified the STING-induced upregulation of genes involved in vascular normalization and endothelial-lymphocyte interaction (Figure 7, E-G) and countervailed the beneficial effects of STING agonist against intratumoral hypoxia (Supplemental Figure 5, C and D). 
A
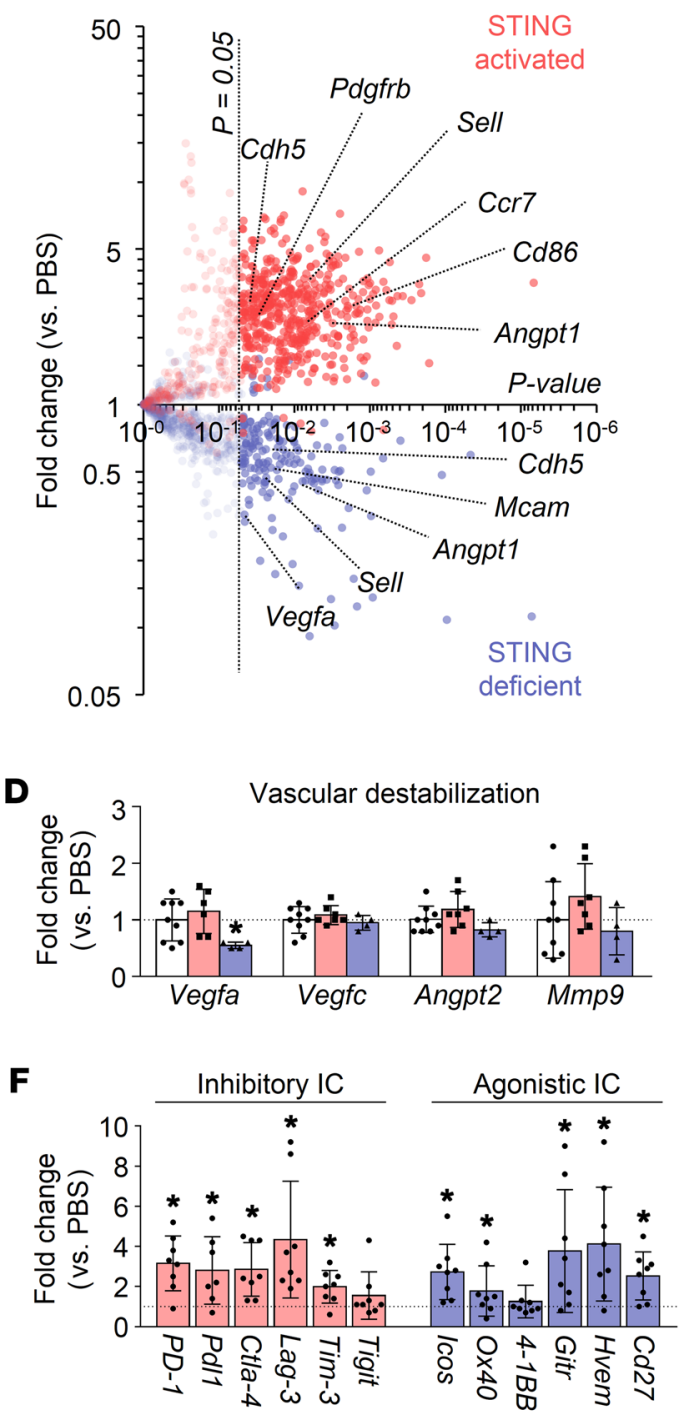

B
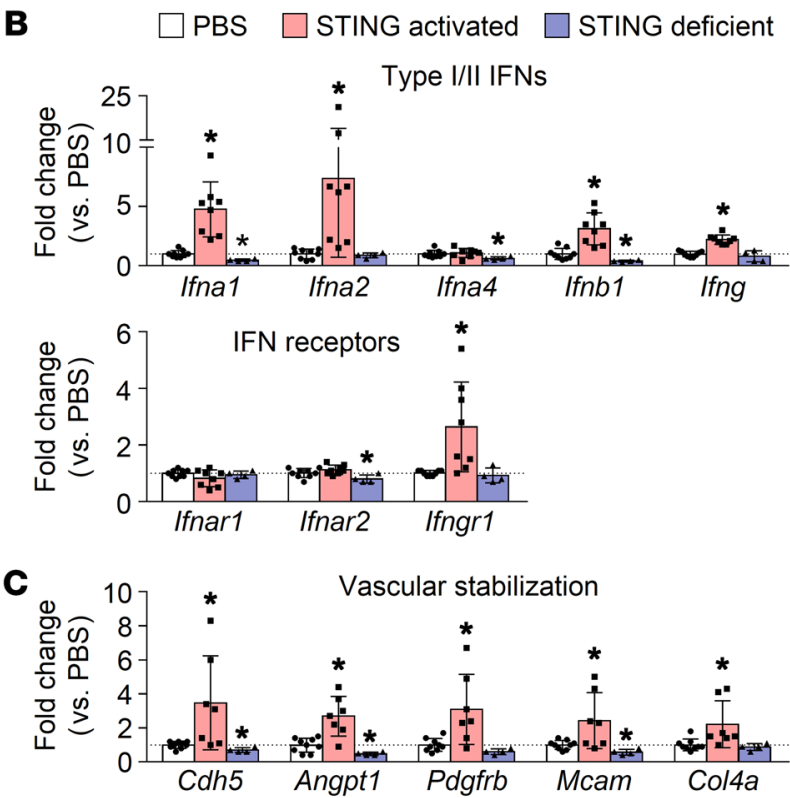

E

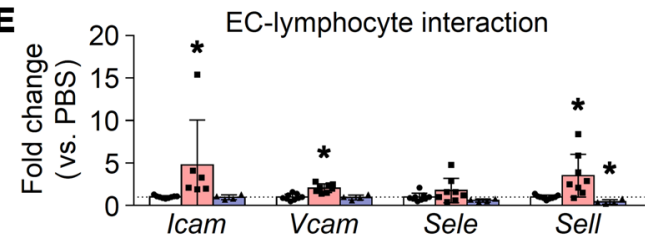

G

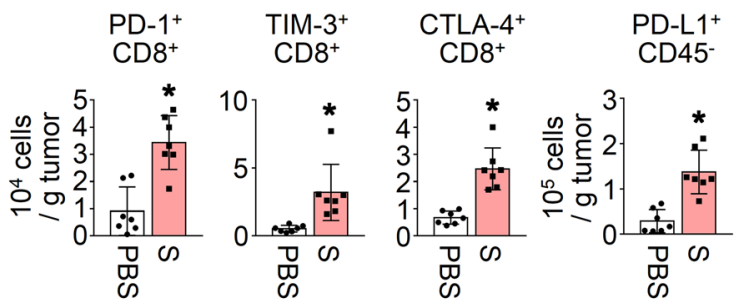

Figure 5. STING signaling pathway regulates tumor vascular and immune microenvironment. WT or STING-deficient mice (STING ${ }^{\text {gt/gt }}$, KO) were injected with LLC tumor cells and treated with intratumoral injections of PBS or STING agonist. (A) Volcano plot showing gene-expression changes in STING agonisttreated tumors of WT mice (red) and PBS-treated tumors of STING-deficient mice (blue). (B-E) Comparison of gene expression related to type I/II IFNs (B), vascular stabilization (C), vascular destabilization (D), and endothelial cell (EC) and lymphocyte interaction (E). (F) Comparison of gene expression related to inhibitory and agonistic immune checkpoints in tumors treated with STING agonist. (G) Flow cytometric analyses of immune checkpoints in tumors. Pooled data from 2 experiments with $n=4$ to 9 per group. Values are shown as mean \pm SD. ${ }^{*} P<0.05$ versus PBS. Two-tailed Student's $t$ test (B-G).

Furthermore, depletion of either IFNAR or CD8 with a neutralizing antibody almost negated the upregulation of M1-specific genes (Supplemental Figure 5, E and F).

These results indicate that type I IFN signaling and $\mathrm{CD} 8^{+} \mathrm{T}$ cells are indispensable for the STING-induced remodeling of tumor vasculatures. Since the impact of IFNAR depletion seems more powerful than that of CD8 depletion and the degree of vascular remodeling mediated by IFNAR and CD8 seems comparable, it seems that IFNAR signaling provides more widespread effects on the immune cells, probably through innate immune cells rather than $\mathrm{CD} 8^{+} \mathrm{T}$ cells.

STING agonist treatment combined with VEGFR2 blockade induces complete tumor regression and enhances vascular normalization in established tumors. Type I IFN signaling is negatively regulated by VEGF signaling (29). Thus, we questioned whether blocking VEGF signaling could further enhance STING-induced type I IFN activation and reinforce STING-induced vascular normalization and antitumor immunity. To explore this combinatorial potential, we examined the effects of VEGFR2 blockade with or without cGAMP treatment in LLC tumors. Treatment with cGAMP and the VEGFR2 antibody DC101 (25 mg/ $\mathrm{kg})$ led to $73 \%$ reduced tumor growth compared with control, which showed $45 \%$ or $61 \%$ reduced tumor growth compared with cGAMP or DC101 monotherapy, respectively (Figure 8, A and B). Combined treatment with cGAMP and DC101 also led to a $47 \%$ reduction in $\mathrm{CD} 31^{+}$blood vascular density and a 1.3 -fold increase in $\mathrm{NG}^{+}$ pericyte coverage compared with cGAMP monotherapy (Figure $8, \mathrm{C}$ and D). Of note, combination treatment with RR-CDA $(25 \mu \mathrm{g}$, twice) instead of cGAMP and DC101 induced complete 
A

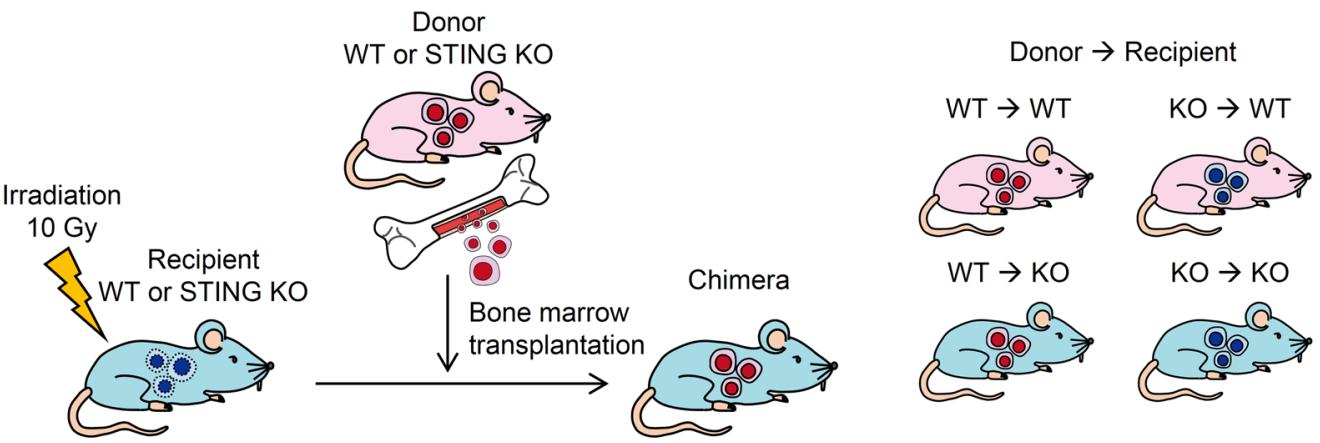

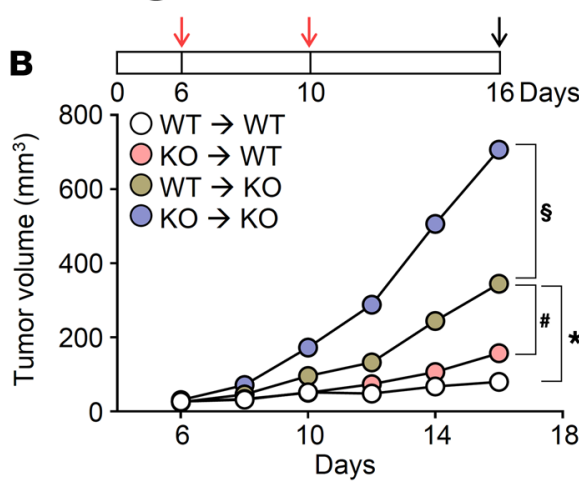

D
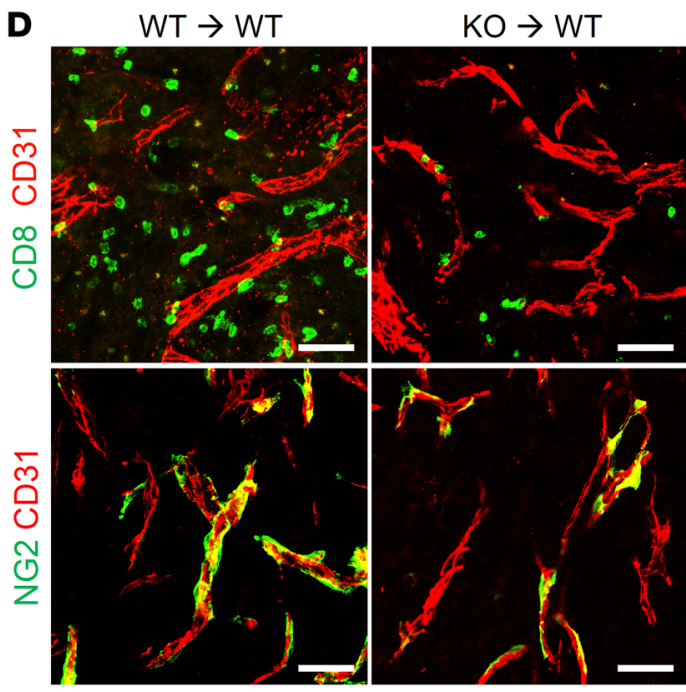
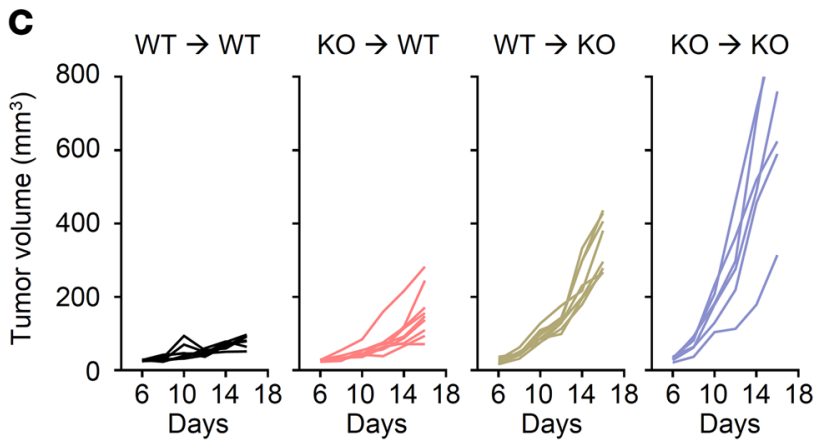
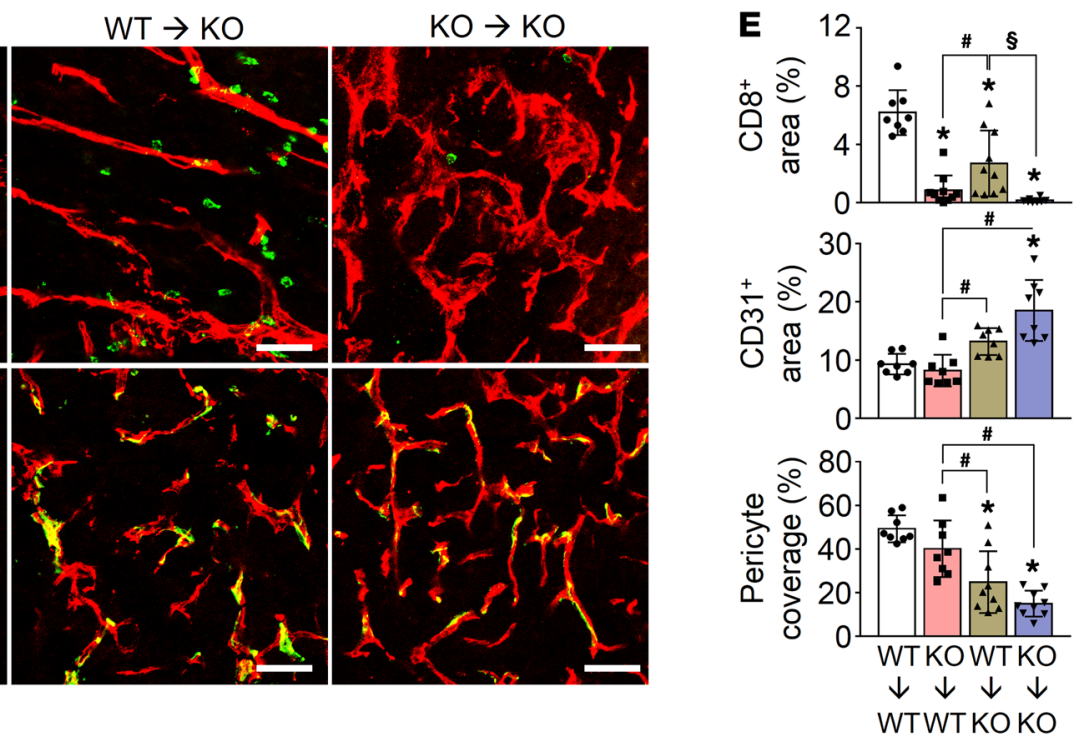

Figure 6. STING in nonhematopoietic cells is as important as STING in hematopoietic cells during therapy with exogenous STING agonist. LLC tumor cells were implanted subcutaneously into bone marrow chimeric mice and treated with intratumoral injections of STING agonist (RR-CDA, $25 \mu$ g). Red arrows indicate treatment, and black arrow indicates sacrifice. (A) Diagram depicting the generation of chimeric mice. (B and C) Comparison of LLC tumor growth in bone marrow chimeric mice. Mean (B) and individual (C) tumor growth curves over time. (D and E) Representative images (D) and comparisons (E) of CD8 ${ }^{+}$T cells, CD31+ blood vessels, and NG2+ pericyte coverage. Pooled data from 2 experiments with $n=6$ to 10 per group. Values are shown as mean \pm SD. ${ }^{*} P<0.05$ versus WT $\rightarrow \mathrm{WT}$; ${ }^{\#} P<0.05$ versus $\mathrm{KO} \rightarrow \mathrm{WT}$; ${ }^{\circledR} P<0.05$ versus WT $\rightarrow$ KO. ANOVA with Tukey's post hoc test (B and E). Scale bars: $50 \mu \mathrm{m}$.

tumor regression in all LLC tumor-bearing mice (Figure 8, E-G). Accordingly, the mice treated with both RR-CDA and DC101 did not die, while the control, DC101 monotherapy, and RR-CDA monotherapy groups had median survival rates of 26, 25, and 38 days, respectively (Figure $8 \mathrm{H}$ ). These results were recapitulated in the CT26 colon cancer model, in which we observed comparable synergistic anticancer effects. Most of the CT26 tumors completely regressed after combination therapy of RR-CDA and
DC101 (Supplemental Figure 6, A and B); these also exhibited similar tumor vessel normalization and increased $\mathrm{CD}^{+} \mathrm{T}$ cell infiltration (Supplemental Figure 6C).

To delineate the mediators of the response to combination therapy of STING agonists and DC101, we depleted either IFNAR, $\mathrm{CD}^{+} \mathrm{T}$ cells, or macrophages. Intriguingly, while neutralization of either IFNAR or CD8 almost completely negated the efficacy of RR-CDA and DC101 treatment and the tumor no 

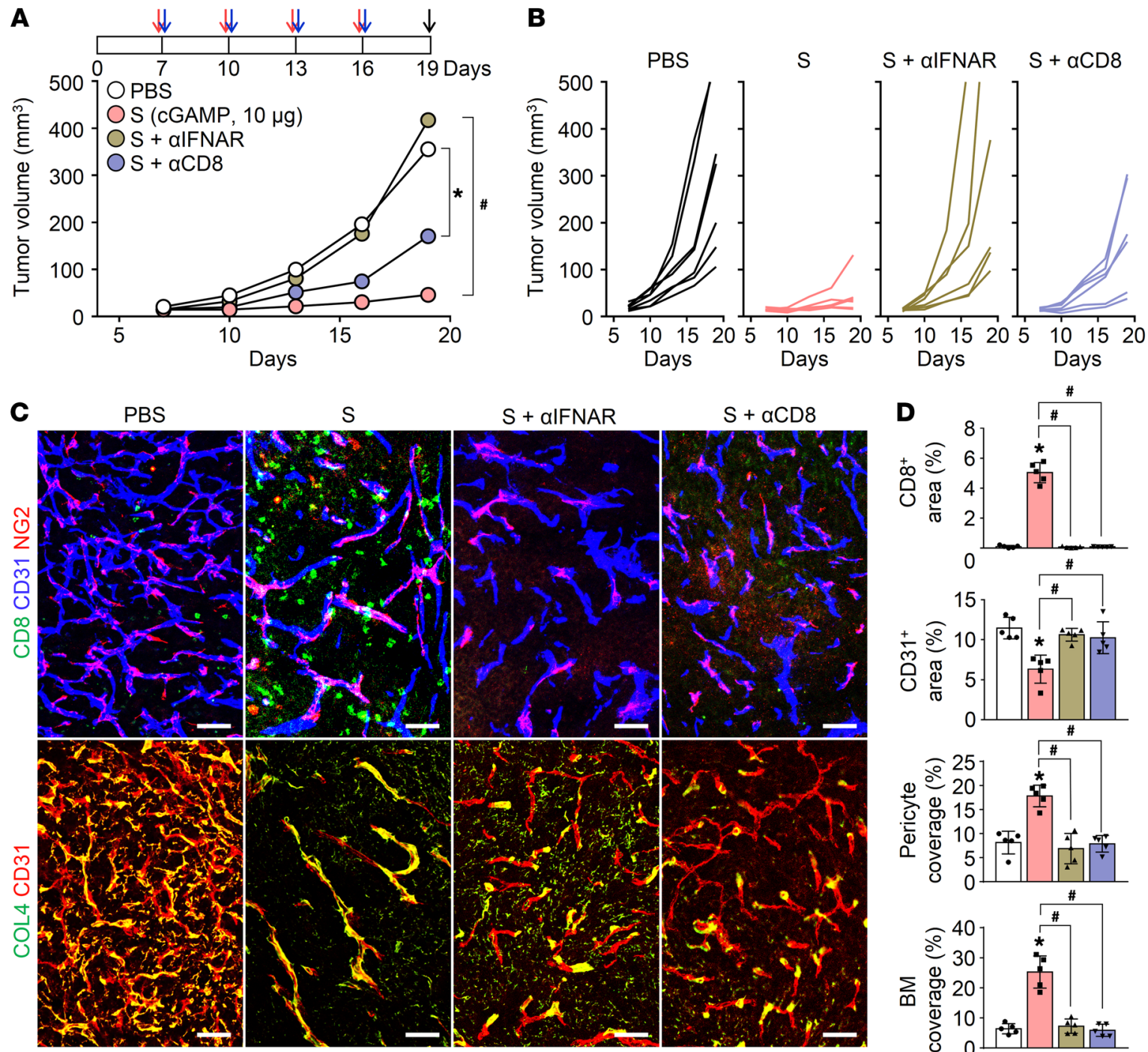

$\mathbf{E}$
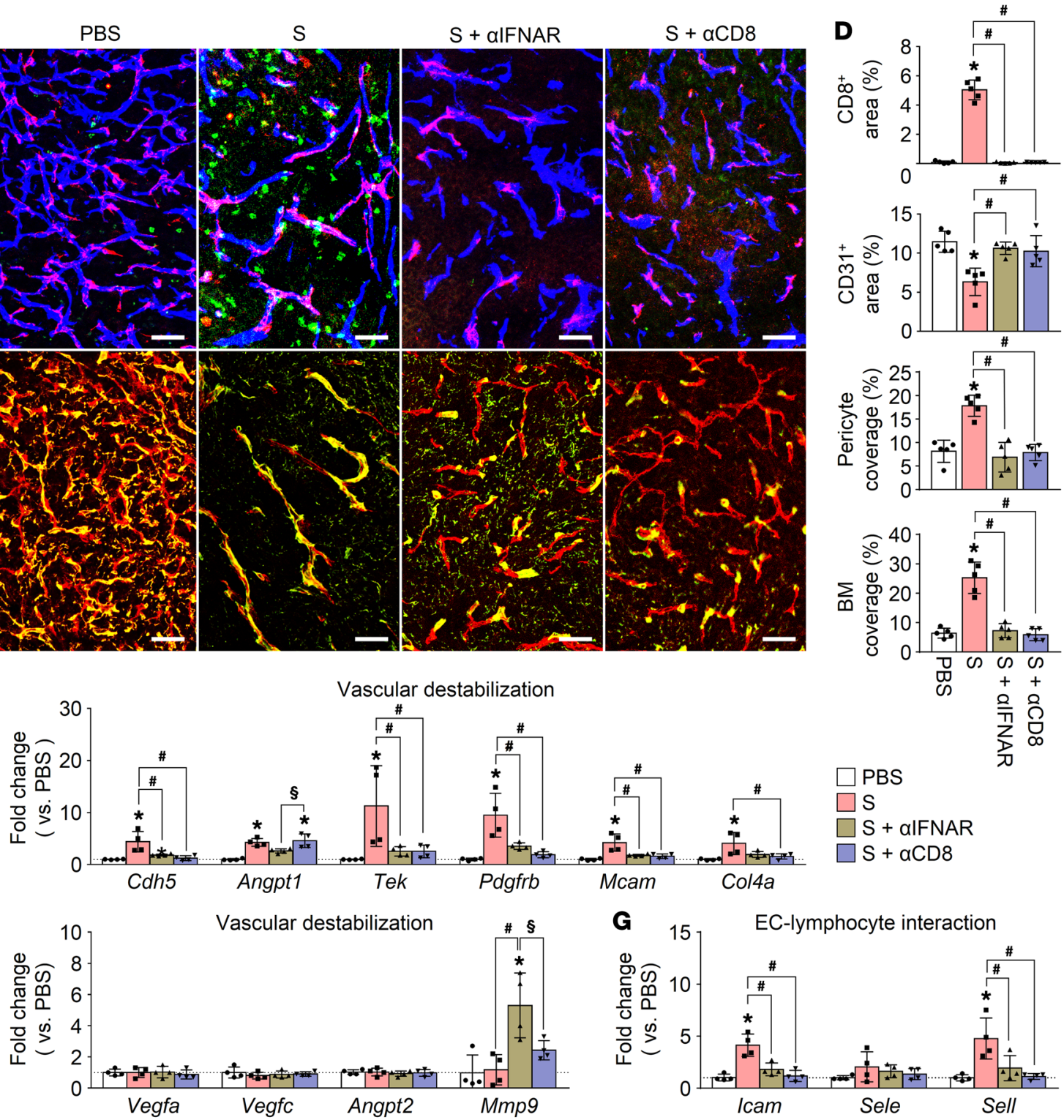

Figure 7. TME regulation by STING activation is dependent on type I IFN signaling and CD8+ $\mathbf{T}$ cells. Mice were subcutaneously implanted with LLC tumor cells and treated with STING agonist (S) and depleting antibodies for IFNAR ( $\alpha$ IFNAR) or CD8 ${ }^{+}$T cells ( $\alpha$ CD8). (A and B) Comparison of tumor growth in mice. Mean (A) and individual (B) tumor growth curves over time. Red arrows indicate injections of cGAMP (10 $\mu$ g), blue arrows indicate injections of depleting antibodies, and black arrow indicates sacrifice. (C and D) Representative images (C) and comparisons (D) of CD8 ${ }^{+} T$ cells, CD31 ${ }^{+}$ blood vessels, NG2+ pericyte coverage, and COL4+ BM coverage. ${ }^{\$} \mathrm{P}<0.05$ versus $\mathrm{S}+\alpha$ IFNAR. (E-C) Comparison of gene expression involved in vascular stabilization (E), vascular destabilization (F), and endothelial-lymphocyte interaction (G). Pooled data from 2 experiments with $n=6$ per group. Values are shown as mean \pm SD. ${ }^{*} P<0.05$ versus PBS; ${ }^{P} P<0.05$ versus S. ANOVA with Tukey's post hoc test (A and D-G). Scale bars: $50 \mu \mathrm{m}$. 
A
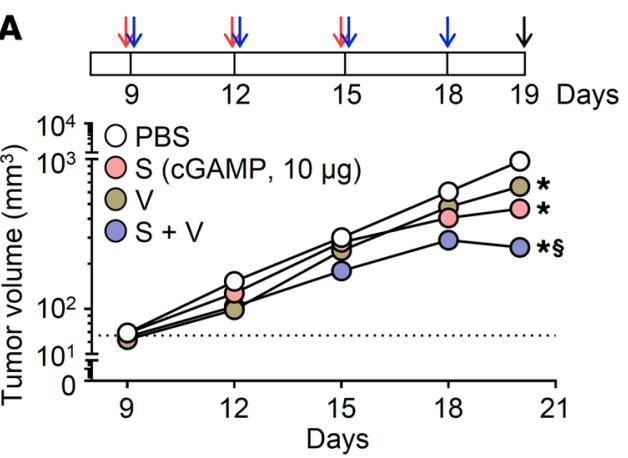

B

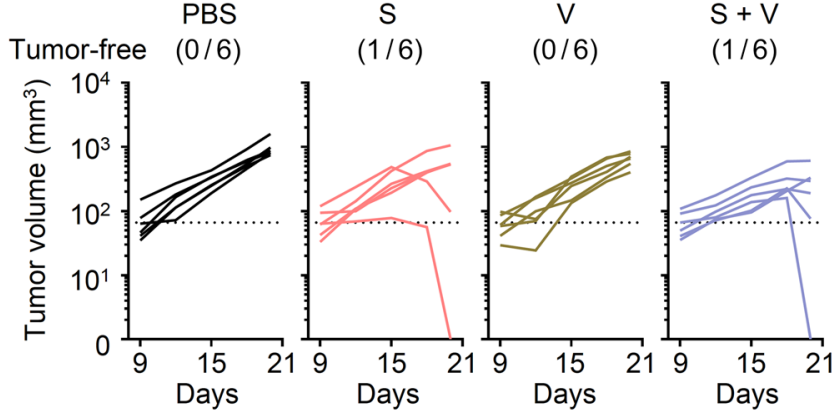

C

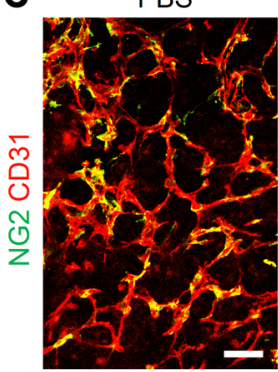

$\mathrm{s}$

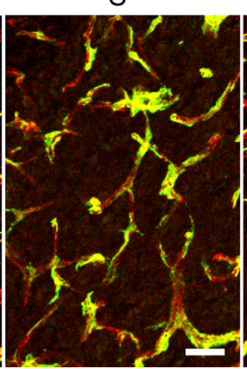

$\mathrm{V}$

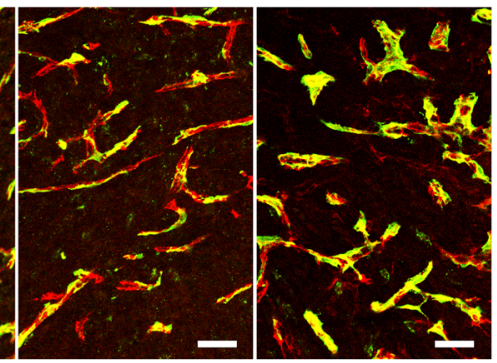

D
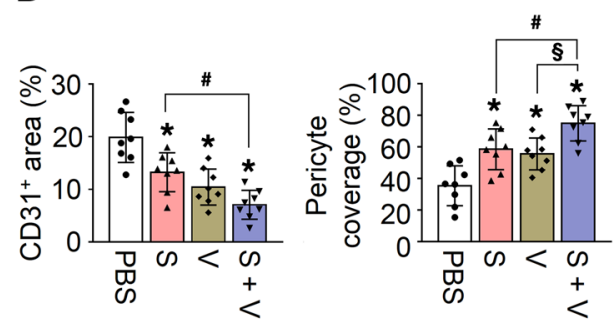

E

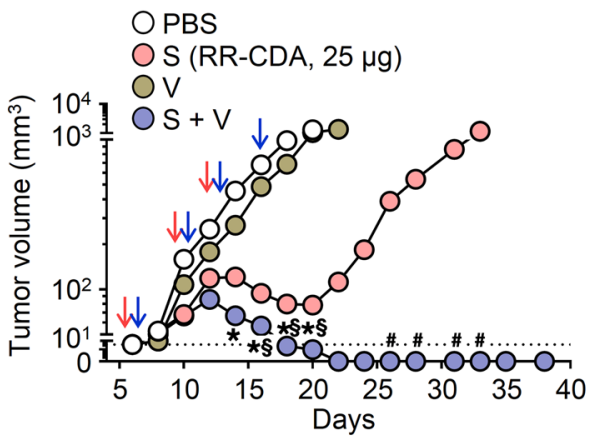

$\mathbf{F}$

Tumor-free $(0 / 8)$

$\mathrm{S}$

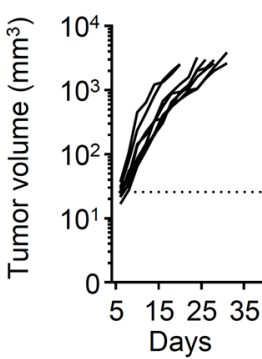

$(3 / 8)$

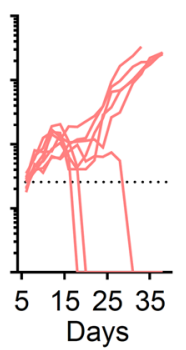

$\mathrm{V}$

$(0 / 8)$
$\mathrm{S}+\mathrm{V}$

$(8 / 8)$

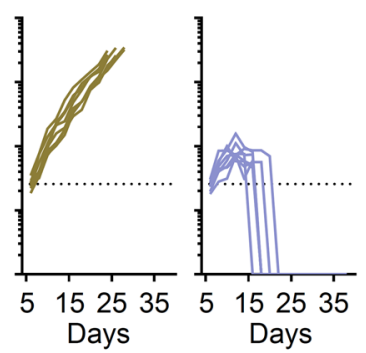

H

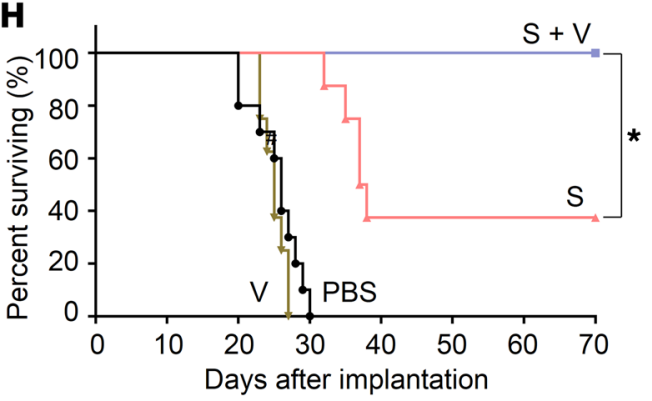

Figure 8. STING agonist treatment combined with VEGFR2 blockade induces complete tumor regression and enhances vascular normalization in established tumors. Mice were subcutaneously implanted with LLC tumor cells and treated with STING agonist (S) and/or DC101 (V). Red arrows indicate injections of STING agonists, blue arrows indicate injections of DC101, and black arrow indicates sacrifice. (A and B) Comparison of LLC tumor growth in mice treated with cGAMP (10 $\mu \mathrm{g})$ and/or DC101. Mean (A) and individual (B) tumor growth curves over time. The number of tumor-free mice is indicated for each group. (C and $\mathbf{D})$ Representative images (C) and comparisons (D) of CD31+ blood vessels and NG2+ pericyte coverage. (E and F) Comparison of LLC tumor growth in mice treated with RR-CDA $(25 \mu \mathrm{g})$ and/or DC101. Mean (E) and individual (F) tumor growth curves over time. The number of tumor-free mice is indicated for each group. (G) Waterfall plots showing the maximal percentage of changes of each tumor at the end of the experiment compared with baseline volume. (H) Kaplan-Meier survival curves for overall survival. ${ }^{*} P<0.05$, log-rank test. Unless otherwise denoted, pooled data from 2 experiments with $n=6$ to 8 per group. Values are shown as mean \pm SD. ${ }^{*} P<0.05$ versus PBS; ${ }^{P} P<0.05$ versus $S ;{ }^{\circledR} P<0.05$ versus V. ANOVA with Tukey's post hoc test $(\mathbf{A}, \mathbf{D}$, and $\mathbf{E})$. Scale bars: $50 \mu \mathrm{m}$.

longer showed complete regression, the antitumor effects of this combination therapy was maintained after the depletion of macrophages (Supplemental Figure 7, A and B).

Taken together, these data show that the combination of STING agonist and VEGFR2 blockade can induce complete tumor regression and durable anticancer immunity, with further enhancement of tumor vascular normalization. Moreover, the efficacy of dual combination therapy of STING agonist and VEGFR2 blockade largely depend on type I IFN signaling and $C D 8^{+} \mathrm{T}$ cells, while macrophages are dispensable. 
A
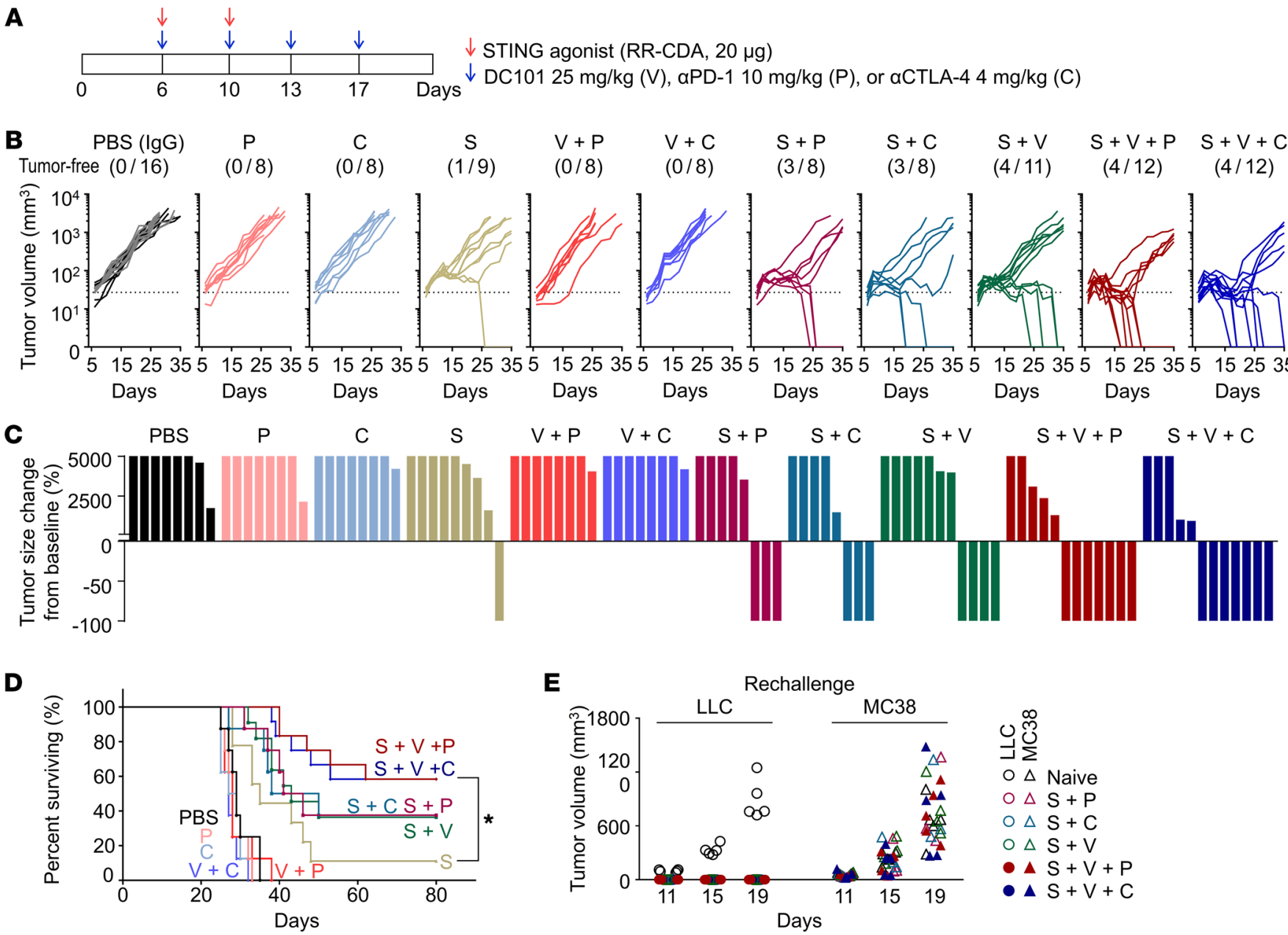

Figure 9. Triple combination immunotherapy of STING agonist, immune checkpoint inhibitor ( $\alpha$ PD-1 or $\alpha$ CTLA-4), and anti-VEGFR2 antibody induces tumor regression. Mice were subcutaneously implanted with LLC tumor cells and treated with STING agonist (S), DC101 (V), and $\alpha$ PD-1 (P) or $\alpha C T L A-4$ (C). (A) Diagram depicting treatment schedule. (B) Comparison of LLC tumor growth in mice. The number of tumor-free mice is indicated for each group. PBS (black) or IgC (gray) was used as control. (C) Waterfall plots showing the maximal percentage changes of each tumor at the end of the experiment compared with baseline volume. (D) Kaplan-Meier survival curves for overall survival. (E) Comparison of tumor growth after injection of LLC or MC38 tumor cells into naive mice or mice with complete tumor regression. Pooled data from 2 experiments with $n=8$ to 16 per group. Values are shown as mean \pm SD. ${ }^{*} P<0.05$, log-rank test (D). ANOVA with Tukey's post hoc test $(\mathbf{E})$.

Triple combination immunotherapy of STING agonist, immune checkpoint inhibitor ( $\alpha P D-1$ or $\alpha C T L A-4)$, and anti-VEGFR2 antibody induces tumor regression. Although STING activation triggered potent antitumor $\mathrm{T}$ cell responses, it also led to a parallel induction of immune checkpoints in the TME, which would presumably generate a negative feedback loop (Figure 5, F and G). Since this could potentially restrain STING -induced anticancer immunity, we evaluated the effects of combining immune checkpoint inhibitors with STING agonist treatment and VEGFR2 blockade to maximize anticancer efficacy (Figure 9A). Since the previous dose of RR-CDA ( $25 \mu \mathrm{g}, 3$ times) already induced complete tumor regression in combination with DC101, its dose was decreased to $40 \mu \mathrm{g}$ (20 $\mu \mathrm{g}$, twice) for the following experiments. Although LLC tumors were completely resistant to immune checkpoint inhibitor ( $\alpha \mathrm{PD}-1$ or aCTLA-4) monotherapy, combining RR-CDA with either $\alpha$ PD-1 $(\mathrm{S}+\mathrm{P})$ or $\alpha$ CTLA-4 $(\mathrm{S}+\mathrm{C})$ improved the antitumor effects compared with monotherapy, showing more than $35 \%$ complete response rates. Furthermore, when LLC tumors were treated with a triple combination immunotherapy with RR-CDA, $\alpha$ VEGFR2, and either $\alpha \mathrm{PD} 1$ or $\alpha \mathrm{CTLA} 4(\mathrm{~S}+\mathrm{V}+\mathrm{P}$ or $\mathrm{S}+\mathrm{V}+\mathrm{C})$, more than half of tumor-bearing mice exhibited complete tumor regression (Figure 9, B and C) and consequently had improved overall survival (Figure 9D). Moreover, we found that the mice that experienced complete regression were immune to rechallenge with LLC tumor cells, but were vulnerable to MC38 tumor cells, suggesting the establishment of long-lasting tumor-specific immunological memory (Figure 9E). In summary, concurrent administration of immune checkpoint inhibitors can counteract STING-induced upregulation of immune checkpoints and potentiate the therapeutic efficacy of STING-targeted cancer immunotherapy, eventually leading to complete tumor regression and long-lasting immune memory against immunotherapy-resistant tumors.

Triple combination immunotherapy efficiently delays tumor growth and suppresses distant metastasis in a spontaneous breast cancer model. Because subcutaneously implanted tumor models have poor and immature vasculatures and lack appropriate tumor 
A

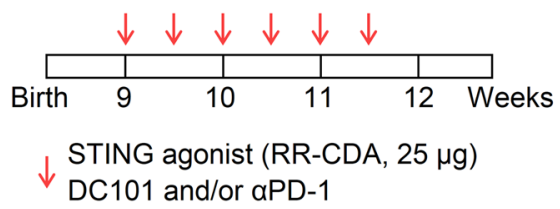

B

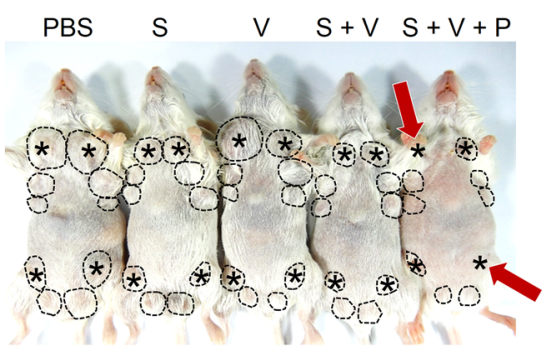

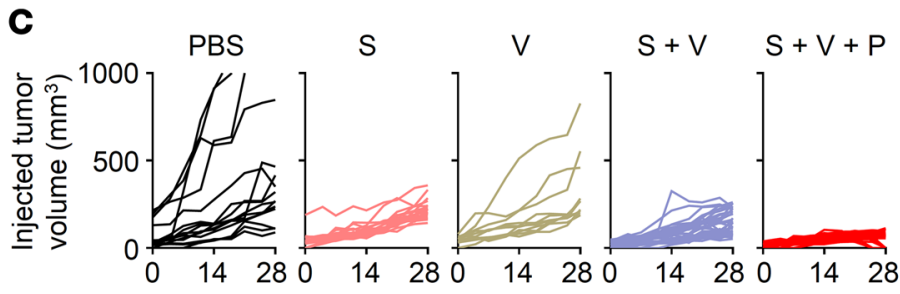

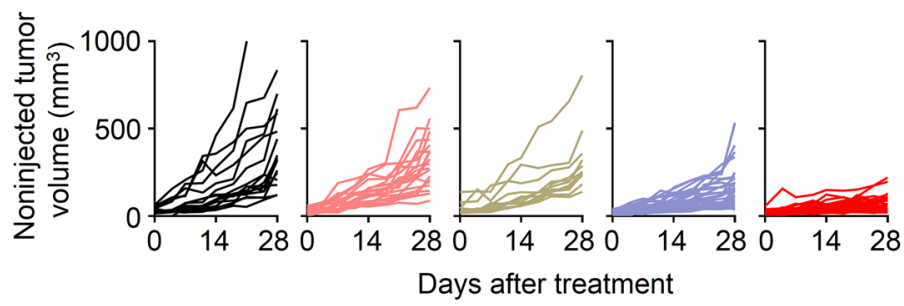

D
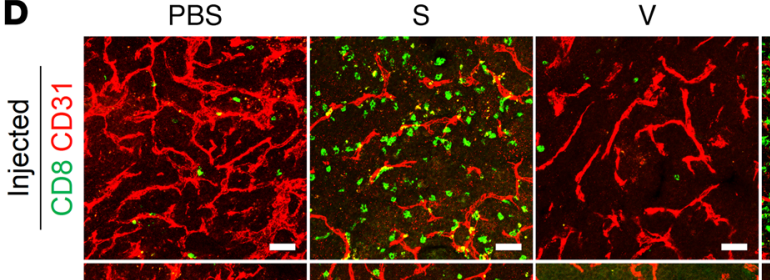

$\mathrm{S}+\mathrm{V}$

$\mathrm{S}+\mathrm{V}+\mathrm{P}$
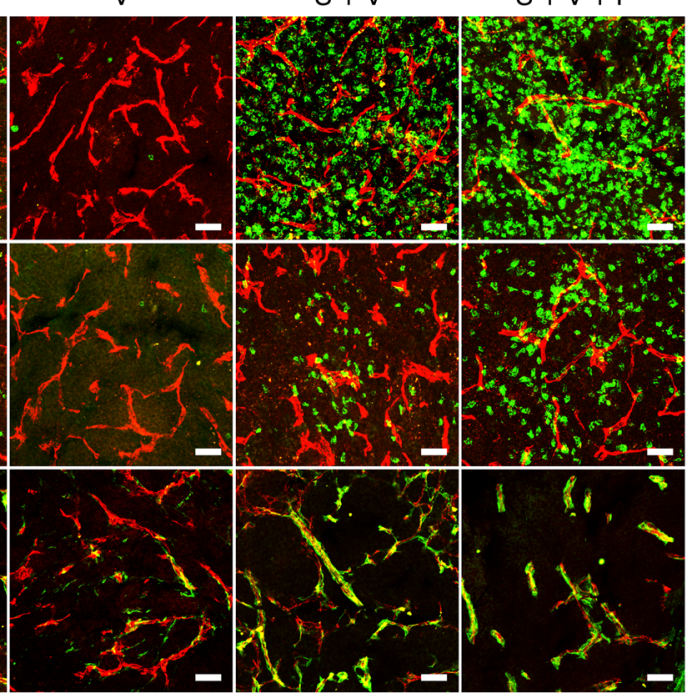
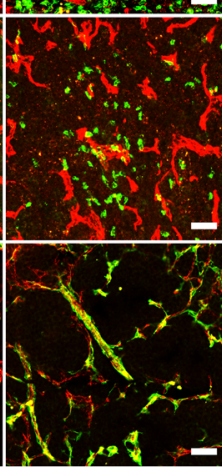
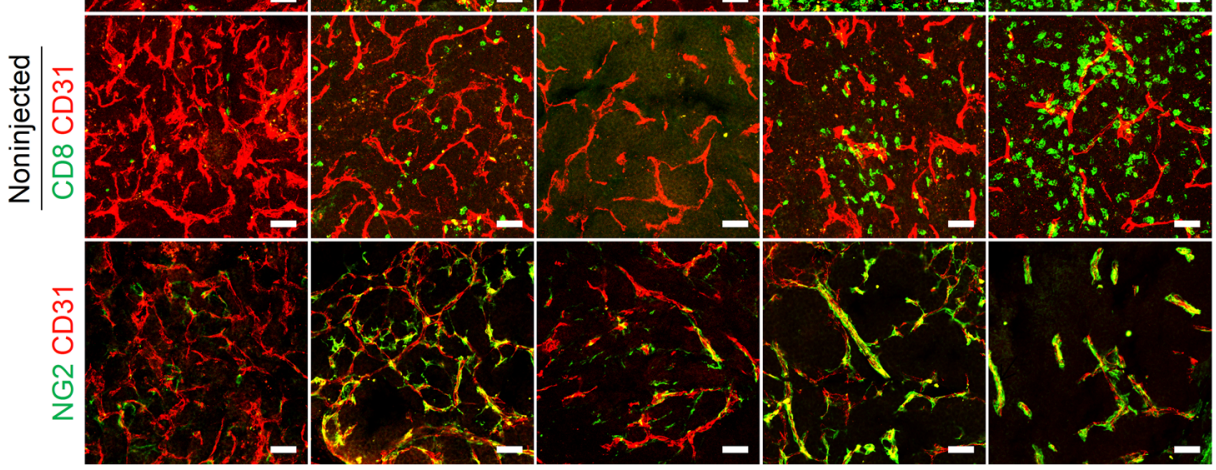

E
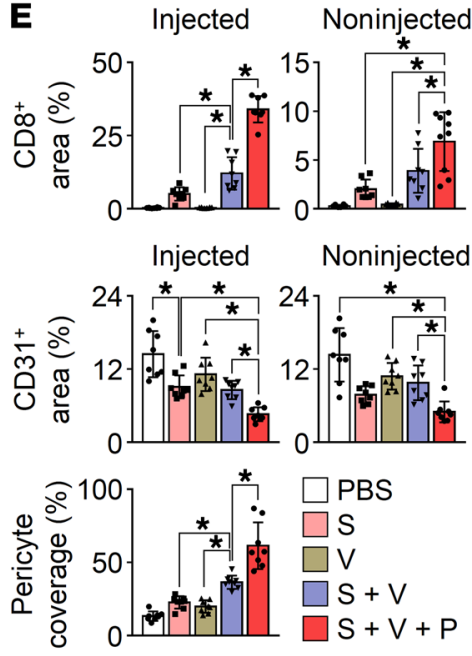

Figure 10. Triple combination immunotherapy efficiently delays tumor growth in both injected and noninjected tumors of a spontaneous breast cancer model. Tumor growth was measured twice a week in a spontaneous breast tumor model, MMTV-PyMT mice, starting from 9 weeks after birth. Mice were treated with STING agonist (S), DC101 (V), and/or $\alpha$ PD-1 (P). (A) Diagram depicting the treatment schedule. Red arrows indicate treatment, and black arrow indicates sacrifice. (B) Representative images showing gross appearances of tumors. Dotted lines demarcate palpable tumor nodules. Asterisks indicate PBS or STING-injected lesion. Red arrows indicate lesions with complete tumor regression. (C) Comparison of the growth of STING-injected or noninjected tumors in MMTV-PyMT mice. ( $\mathbf{D}$ and E) Representative images (D) and comparisons (E) of CD8 ${ }^{+}$T cells, CD31+ blood vessels, and NG2 ${ }^{+}$pericyte coverage. $n=$ 5 to 7 per group. Values are shown as mean \pm SD. ${ }^{*} P<0.05$, ANOVA with Tukey's post hoc test (E). Scale bars: $50 \mu \mathrm{m}$.

stroma, they may not fully represent the biology of a real tumor immune microenvironment. Therefore, we employed a spontaneous breast cancer model, MMTV-PyMT, which has more mature tumor vasculatures and abundant stromal cells and is therefore a reliable representative of human breast cancer $(30,31)$, to further validate the efficacy of STING activation in combination with VEGFR2 blockade and immune checkpoint inhibition (Figure 10A). After 3 weeks of treatment, RR-CDA alone remarkably delayed tumor growth, not just in the STING-injected tumor, but also in the noninjected tumors, suggesting abscopal antitumor effects upon STING activation; this was further strengthened by adding VEGFR2 blockade $(\mathrm{S}+\mathrm{V})$, and the triple combination therapy of RR-CDA, DC101, and anti-PD1 antibody (S + V + P) displayed the most potent tumor growth inhibition effect (Figure 10, B and C). Similarly to our previous observations, both dual and triple combination therapy led to a remarkable increase in intratu- moral $\mathrm{CD} 8^{+} \mathrm{T}$ cell infiltration, a decrease in tumor vessel density, and enhanced pericyte coverage (Figure 10, D and E). Moreover, triple combination immunotherapy markedly reduced hematogenous lung metastases (Figure 11, A and B) and prolonged the overall survival of MMTV-PyMT mice (Figure 11C). Taken together, these results demonstrate that STING agonist treatment, combined with VEGFR2 and PD-1 inhibition, can effectively inhibit tumor progression and metastasis through vascular normalization and bolster anticancer immune response (Figure 11D).

\section{Discussion}

Our present findings demonstrate the importance of STING signaling in tumor vascular reprogramming and support the clinical applicability of STING agonists for antiangiogenic immunotherapy in cancer treatment. Vascular STING expression in tumor tissue correlated with favorable prognosis in human malignancies, 
A

A

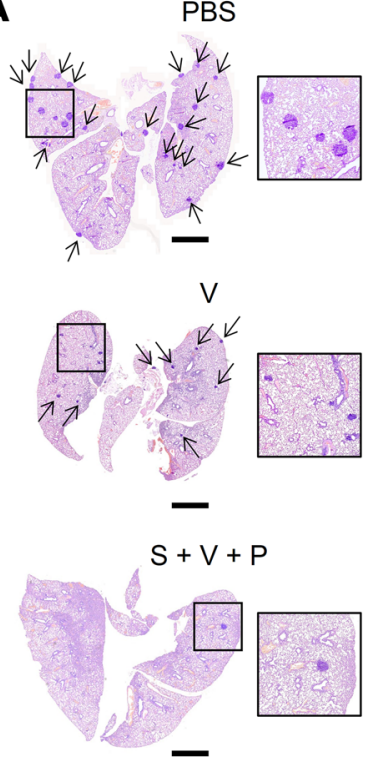

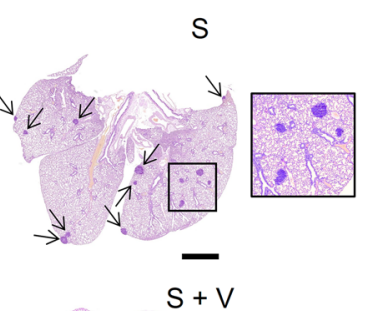

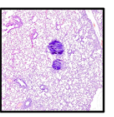

B

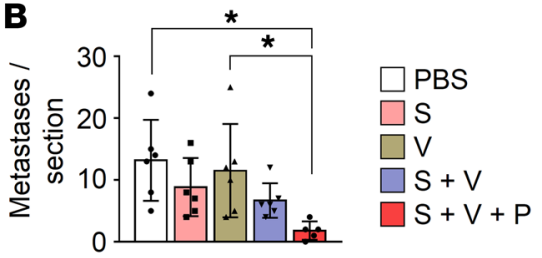

C

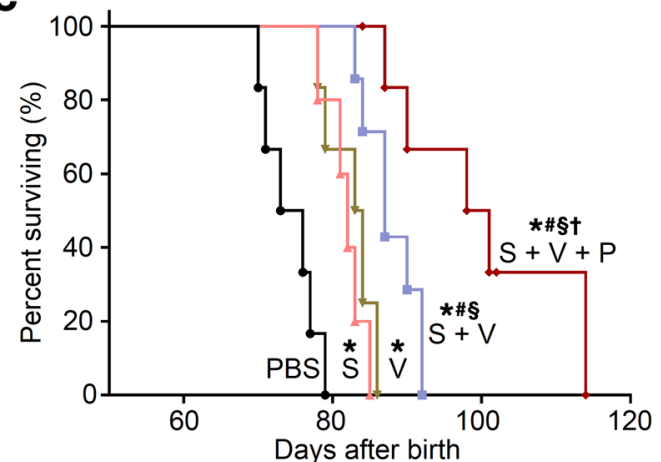

D
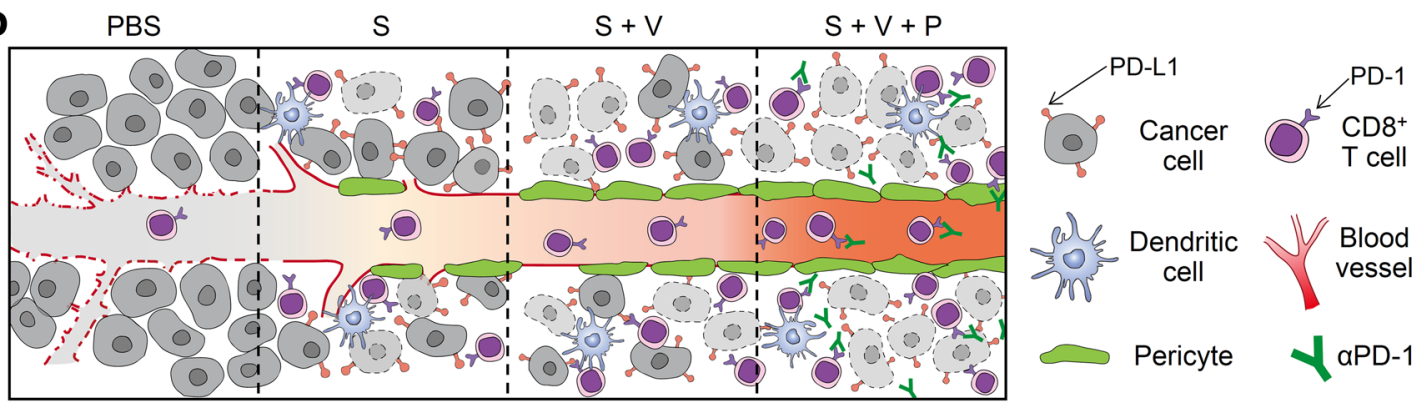

Figure 11. Triple combination immunotherapy suppresses lung metastases and provides survival benefit in a spontaneous breast cancer model. Tumor growth was measured twice a week in a spontaneous breast tumor model, MMTV-PyMT mice, starting from 9 weeks after birth. Mice were treated with STING agonist (S), DC101 (V), and/or $\alpha$ PD-1 (P). (A) Lung sections stained with H\&E. Arrows indicate pulmonary metastatic lesions. Scale bars: 2 mm. $n=$ 5 to 6 per group. (B) Comparison of the number of metastatic colonies per lung section. Values are shown as mean \pm SD. ${ }^{*} P<0.05$, ANOVA with Tukey's post hoc test. (C) Kaplan-Meier survival curves for overall survival. $n=5$ to 7 per group. Values are shown as mean \pm SD. ${ }^{*} P<0.05$ versus $P B S$; $P<0.05$ versus $\mathrm{S} ;{ }^{\S} P<0.05$ versus $\mathrm{V} ;{ }^{\dagger} P<0.05$ versus $\mathrm{S}+\mathrm{V}$, log-rank test. (D) Diagram depicting the mechanism by which STING activation reprograms TME and the rationale for STING-based combination immunotherapy.

and intratumoral activation of STING signaling by exogenous STING agonists induced quantitative and qualitative changes of tumor vasculature. Upon STING activation, tumor vessels underwent a transient phase of initial vascular disruption and then became normalized, with enhanced pericyte coverage and upregulation of endothelial-leukocyte adhesion molecules. This process facilitated the intratumoral trafficking of effector $\mathrm{T}$ cells across the endothelial barrier and conditioning of the TME to enhance antitumor immunity. Most importantly, simultaneous blockade of VEGFR2 and/or immune checkpoint molecules (PD-1 or CTLA-4) amplified the antitumor efficacy of STING agonists, promoting complete tumor regression and prolonged survival in mice with tumors that were resistant to immunotherapy or antiangiogenic therapy alone.

STING-induced vascular remodeling was found to involve multiple TME components that are closely intertwined with each other. Most importantly, type I IFNs were indispensable mediators of STING-induced vascular reprogramming. IFN- $\beta$ is a potent antiangiogenic cytokine that inhibits endothelial proliferation, survival, and capillary network formation, which induces vascular maturation in tumors through angiopoietin-1 upregulation (32-34). Consistently, our findings revealed that intratumoral STING agonist treatment led to the activation of type I IFN signaling accompanied by upregulation of vascular normalizing genes, such as Angpt1, Pdgfrb, Mcam, Cdh5, and Col4a. These transcriptional changes eventually induced tumor vessel normalization with enhanced pericyte coverage and more intact basement membrane, which promoted intratumoral infiltration of effector $\mathrm{CD}^{+} \mathrm{T}$ cells and alleviated hypoxia in the TME. Notably, blocking type I IFN signaling by IFNAR inhibition almost completely abrogated STING-induced transcriptional alterations and vascular phenotypical changes, indicating a critical role for type I IFNs in STING-induced vascular reprogramming.

Another important component of STING-induced vascular reprogramming is nonhematopoietic stromal cells, such as endothelial cells. As revealed through our bone marrow chimera experiments, STING in nonhematopoietic cells is as important as STING in hematopoietic cells for eliciting maximal therapeu- 
tic efficacy of exogenous STING agonists. Especially, STING in nonhematopoietic cells seems to be more important in antiangiogenic and vascular normalizing effects of STING agonists compared with STING in hematopoietic cells. Among various stromal cell components in TME, endothelial cells seem to be the key players after STING activation for several reasons. First, they show the most distinct STING expression compared with other stromal cells. Moreover, they can produce excess IFN- $\beta$ upon stimulation with STING agonists, and in turn, IFN- $\beta$ can directly act on endothelial cells, presumably in an autocrine or paracrine manner $(6,23)$. Furthermore, endothelial cells may not only be the dominant source of type I IFNs, but also their most important target in TME, as they are more abundant than dendritic cells and most tumors are highly angiogenic (23). However, the definite role of endothelial cells and other stromal components of TME should be deciphered through further studies using celltype-specific conditional KO mice.

Innate immune cells are universally found within TME and are also involved in the STING-mediated remodeling of TME. For this process, the most important cell type seems to be dendritic cells, since they produce IFN- $\beta$ robustly upon stimulation with STING agonists and the activation of type I IFN signaling in the dendritic cells is critical for $\mathrm{CD}^{+} \mathrm{T}$ cell priming against tumor antigens (6, 35). Another type of abundant innate immune cells within TME is macrophages. Environmental signals cause macrophages to undergo reversible phenotypic switching into either M1- or M2-like macrophages, which are proposed to suppress and promote tumor angiogenesis, respectively $(13,36,37)$. Our results indicated that intratumoral STING activation preferentially upregulated M1-specific genes and accumulated M1-like macrophages within TME. These phenotypic changes in macrophages seem to be consequences rather than causes of STING agonist-mediated remodeling of TME, given that the depletion of macrophages did not attenuate the efficacy of STING agonists. However, because the macrophages do not seem to be completely depleted by clodronate liposome, it would be difficult to completely rule out the possible contribution of macrophages in STING-induced antitumor activity.

Tumor-infiltrating T lymphocytes also play an important role in the TME and were found to be involved in STING-mediated vascular remodeling. Antiangiogenic therapy strengthens T cellmediated antitumor immunity by enhancing lymphocyte infiltration into the tumor, and growing evidence suggests the reciprocal effects of $\mathrm{T}$ lymphocytes on tumor vasculatures $(15,22,38)$. CD ${ }^{+}$ cytotoxic T cells and Th1 cells secrete IFN- $\gamma$, which inhibits tumor angiogenesis by restraining endothelial cell proliferation and upregulating cytokine-encoding genes (e.g., CXCL9, CXCL10, and CXCL11) that stimulate pericyte recruitment $(13,18)$. Moreover, IFN- $\gamma$ primarily causes regression of immature tumor endothelial cells that are devoid of pericytes, while those with pericytes can endure IFN- $\gamma$-mediated vascular damage (39). Therefore, tumor-infiltrating T cells that secrete IFN- $\gamma$ may also contribute to vascular and immune remodeling.

Monotherapy with a STING agonist exhibited outstanding antitumor efficacy through vascular normalization and $\mathrm{T}$ cell influx to the TME in vivo. However, this could be limited by opposing mechanisms $(40,41)$. Type I IFN signaling is negatively regulated by VEGF/VEGFR2 signaling through ubiquitin-mediated
IFNAR degradation $(29,34)$, such that STING agonist treatment may not sufficiently activate type I IFN signaling in VEGF-rich cancers. Additionally, while repeated STING agonist injections induced strong innate and adaptive immunity, this would inevitably upregulate immune checkpoint molecules, generating a negative feedback loop that might confer adaptive resistance to STING agonist-induced immune responses, ultimately enabling tumor relapse (42-44). This suggests that a combination strategy targeting VEGF/VEGFR2 signaling and/or immune checkpoint signaling might be needed to maximize and sustain the anticancer efficacy of STING-based immunotherapy, especially in the clinical setting in which patients will be repeatedly exposed to STING agonists over a long time period. Indeed, our findings demonstrated that triple combination immunotherapy, with STING agonist, anti-VEGFR2 antibody, and either anti-PD-1 or anti-CTLA-4 antibody, yielded superior and durable antitumor efficacy compared with monotherapy or dual combination therapy. Future clinical trials should investigate and validate this triple combination strategy, especially for patients with poorly immunogenic and highly angiogenic tumors that do not respond to conventional immune checkpoint blockade.

Intratumoral delivery of immunostimulatory drugs has an advantage of achieving much higher concentrations of drugs and immunostimulatory products in TME than does systemic administration. Moreover, it can sometimes induce abscopal effects, the regression of untreated tumors at a distant site, which enables the effective control of distant metastases (45). Though abscopal effects could be induced considerably in preclinical mouse cancer models, it is limited in cancer patients with multiple systemic metastases because the human immune system is more complex and extensive than that of mice. For this reason, many studies tried to enhance the abscopal effect of intratumoral immunotherapy by combining with a systemic treatment (45). In our study with the MMTV-PyMT spontaneous breast model, which has multiple synchronous breast cancer nodules and lung metastases, intratumoral injection of STING agonist successfully delayed the growth of both injected and noninjected tumors. Intriguingly, the degree of abscopal effect was further strengthened with simultaneous systemic treatment of VEGFR2 and PD1 blockade, which led to higher accumulation of $\mathrm{CD} 8^{+} \mathrm{T}$ cells into the tumors. Therefore, simultaneous VEGFR2 blockade could overcome the limitations of intratumoral immunotherapy and enhance the efficacy of STING therapy in future clinical trials.

In addition to its promising efficacy, STING-based triple combination immunotherapy also has the advantage of minimizing systemic treatment-related toxicities. Combination immunotherapies with anti-PD-1 and anti-CTLA-4 antibodies already show superiority over monotherapy in various malignancies, but sometimes lead to fatal toxicities, particularly caused by synergistic hyperactivation of systemic immunity $(46,47)$. In contrast, STING-based immunotherapy can minimize such systemic toxicities because STING agonists are delivered intratumorally and not systemically. In our study, we observed no treatment-related gross abnormalities or lethality in the tumor-bearing animals treated with STING-based triple combination therapy. There remains the risk that intratumoral injection of STING agonists may cause local injection-site reactions. Previous studies report ulcerative or 
hemorrhagic changes of tumors following treatments with various STING agonists, which are reportedly caused by TNF- $\alpha$ secreted from STING-activated myeloid cells $(6,8,11)$. We also observed hemorrhagic crusts covering the tumor masses of regressing tumors after STING-based triple immunotherapy. However, these skin lesions were self-limited and completely resolved within approximately 2 to 3 weeks of treatment. This suggests that STING-based triple immunotherapy will be generally well tolerated, except for the self-limited injection site reaction.

In conclusion, our results demonstrate that intratumoral STING activation normalizes tumor blood vessels and enhances antitumor immunity. STING agonist treatment activates type I IFN signaling, and it yields the most potent antitumor efficacy when combined with VEGFR2 and/or immune checkpoint blockades. These findings warrant further investigation of this combination strategy of STING-based immunotherapy in clinical trials.

\section{Methods}

Mice and cell lines. Male C57BL/6 or BALB/c mice 6 to 8 weeks of age were obtained from Orient Bio Inc. Male C57BL/6J-Tmem173 $3^{\text {gt }} / \mathrm{J}$ mice (STING ${ }^{\mathrm{gt} / \mathrm{gt}}$ ) (catalog 017537) and female MMTV-PyMT transgenic mice (FVB/N) (catalog 002374) were purchased from Jackson Laboratory. All mice were housed in a specific pathogen-free animal facility at CHA University. LLC and CT26 colon cancer cells were purchased from ATCC. MC38 colon cancer cells were obtained from the National Cancer Center (Goyang, South Korea). These cells were cultured at $37^{\circ} \mathrm{C}$ under $5 \% \mathrm{CO}_{2}$ in DMEM supplemented with $10 \%$ FBS and $1 \%$ penicillin/streptomycin.

Tumor models and treatment regimens. We implanted $5 \times 10^{5} \mathrm{LLC}$ and $2 \times 10^{5} \mathrm{CT} 26$ cells into the right flanks of C57BL/6 and BALB/c mice, respectively. When the tumors reached more than $4-5 \mathrm{~mm}$ in diameter, we performed intratumoral injections of the STING agonist 3'3'-cGAMP (10 $\mu \mathrm{g}$, cGAMP, Invivogen) or 2'3'-c-di-AM(PS) ${ }_{2}(25 \mu \mathrm{g}$, RR-CDA, also called MIW815 or ADU-S100, Invivogen) at the given time points. Mice in the control group were intratumorally injected with the same volume of PBS. For combination therapy, we also performed intraperitoneal injections of VEGFR2-blocking antibody (25 $\mathrm{mg} / \mathrm{kg}$, clone DC101, BioXCell) at given time points. For the celldepletion study, each mouse received intraperitoneal injections of 200 $\mu \mathrm{g}$ anti-IFNAR (clone MAR1-5A3, BioXCell), anti-CD8 (clone 53-6.72, BioXCell), anti-IFN- $\gamma$ (clone XMG1.2, BioXCell), or $500 \mu \mathrm{g}$ antiTNF- $\alpha$ (clone XT3.11, BioXCell) antibody every 3 days. For the macrophage cell-depletion study, each mouse received an intraperitoneal injection of $200 \mu \mathrm{l}$ clodronate liposome or control liposome (Liposoma) 3 or 4 times, as described previously (28). For immune-checkpoint blockade, the mice received intraperitoneal injection of anti-PD-1 (10 $\mathrm{mg} / \mathrm{kg}$, clone J43, BioXCell) or anti-CTLA-4 (4 mg/kg, clone 9D9, BioXCell) antibody at the given time points. The surviving mice with complete tumor regression were rechallenged with $5 \times 10^{5} \mathrm{LLC}$ cells or MC38 cells in the left flank, and the tumor growth was monitored. The tumor volumes were measured with calipers and calculated using the following modified ellipsoid formula: $1 / 2 \times\left(\right.$ length $\times$ width $\left.^{2}\right)$. For survival analysis, the mice were euthanized once the tumors exceeded $2500 \mathrm{~mm}^{3}$ in volume or when the mice became moribund. MMTVPyMT mice that were 9 weeks old were administered with intratumoral injections of STING agonist ( $25 \mu \mathrm{g} /$ nodule, RR-CDA) on 4 nodule sites per mouse and intraperitoneal injections of anti-VEGFR2 and/or anti-PD-1 antibodies twice per week for 3 weeks. For survival analysis, the mice were euthanized once the tumor volume exceeded $1500 \mathrm{~mm}^{3}$ or when the mice became moribund.

Analyses of human tumor samples. The survival outcomes and clinicopathologic data were obtained from the electronic medical records of CHA Bundang Medical Center. Overall survival was defined as the time interval between the diagnosis of cancer and death or last follow-up. Immunohistochemical staining was performed using antibodies against STING (rabbit, clone D1V5L, Cell Signaling) or CD8 (rabbit, clone SP57, Roche) on $5 \mu \mathrm{m}$ thick sections cut from formalin-fixed, paraffin-embedded tissue microarray blocks of $3 \mathrm{~mm}$ core size. The visualization systems used were the BenchMark XT (Ventana) with heat-induced epitope retrieval (CC1 solution, Ventana) and the iView DAB Detection Kit (Ventana). Before mounting, the slides were counterstained with hematoxylin. High-resolution digital images of the stained slides were acquired using a BX43 microscope (Olympus). Lymphovascular invasion was defined as the presence of tumor cells within a vascular lumen that are intimately admixed with blood cells and focally adherent to or within the vessel wall. Density measurement of STING or CD8 for every stained core of tissue microarray was performed with ImageJ software running the Fiji image processing package (https://imagej.net/Fiji). The color channels with hematoxylin and diaminobenzidine were separated and quantified to determine the immunoreactive areas. Particularly, the degree of endothelial STING expression was calculated as the mean perimeter of STING positivity around the entire circumference of 10 or more representative tumor blood vessels per core. The cut-off values for high and low STING expression were the median of all the samples of the corresponding cancer type.

For further information, see Supplemental Methods.

Statistics. Statistical analyses were performed using GraphPad Prism 7.0 software (GraphPad Software) and PASW statistics 18 (SPSS). Values are presented as mean \pm SD unless otherwise indicated. The statistical differences were assessed using unpaired 2-tailed Student's $t$ test, $\chi^{2}$ tests, or ANOVA with Tukey's post hoc test. Pearson's correlation analysis was performed to investigate the relationship between $\mathrm{CD}^{+} \mathrm{T}$ cell expression and endothelial STING expression. Waterfall plots present the maximal percentage changes of each tumor at the end of the experiment compared with their baseline volume. Survival curves were generated using the Kaplan-Meier method, and statistical differences were analyzed with the log-rank test. Multivariate analyses for overall survival were conducted with Cox's proportional hazard model. Statistical significance was set at $P<0.05$.

Study approval. The animal experiments were performed according to the animal experimental guidelines of CHA University and with the approval of the Institutional Animal Care and Use Committee of CHA University (IACUC 150080). All human samples were collected with informed consent from patients at CHA Bundang Medical Center. All procedures were approved by the institutional review board of CHA Bundang Medical Center (IRB no. 2017-11-054).

\section{Author contributions}

HY, WSL, HJC, and CK were responsible for the study concept and design. HY, WSL, SJK, JHK, SKC, SK, GK, HJC, and CK conducted the experiments. HY, WSL, CGK, HJC, and CK performed data analysis. HY, HJC, and CK generated the figures and wrote and reviewed the manuscript. CK and HJC supervised the study, 
obtained funding, and drafted the manuscript. Authorship order between HJC and CK was determined based on greater responsibility for supervision, funding, and manuscript writing

\section{Acknowledgments}

This study was supported by the National Research Foundation of Korea (NRF) grant funded by the Korean government (MSIT) (NRF-2016R1C1B2014671 to CK) and by the Bio \& Medical Tech- nology Development Program of the National Research Foundation (NRF-2016M3A9E8941664 to HJC).

Address correspondence to: Chan Kim or Hong Jae Chon, Medical Oncology, CHA Bundang Medical Center, CHA University Medical School, 59 Yatap-ro, Bundang-gu, Seongnam, 13496, Korea. Phone: 82.31.881.7588; Email: chan@cha.ac.kr (CK); Phone: 82.31.780.3737; Email: minidoctor@cha.ac.kr (HJC).
1. Woo SR, et al. STING-dependent cytosolic DNA sensing mediates innate immune recognition of immunogenic tumors. Immunity. 2014;41(5):830-842.

2. Barber GN. STING: infection, inflammation and cancer. Nat Rev Immunol. 2015;15(12):760-770.

3. Gajewski TF. The next hurdle in cancer immunotherapy: overcoming the non-T cell-inflamed tumor microenvironment. Semin Oncol. 2015;42(4):663-671

4. Corrales L, Gajewski TF. Molecular pathways: targeting the stimulator of interferon genes (STING) in the immunotherapy of cancer. Clin Cancer Res. 2015;21(21):4774-4779.

5. Rivera Vargas T, Benoit-Lizon I, Apetoh L. Rationale for stimulator of interferon genes-targeted cancer immunotherapy. Eur JCancer. 2017;75:86-97.

6. Corrales L, et al. Direct activation of STING in the tumor microenvironment leads to potent and systemic tumor regression and immunity. Cell Rep. 2015;11(7):1018-1030.

7. Fu J, et al. STING agonist formulated cancer vaccines can cure established tumors resistant to PD-1 blockade. Sci Transl Med. 2015;7(283):283ra52.

8. Ager CR, Reilley MJ, Nicholas C, Bartkowiak T, Jaiswal AR, Curran MA. Intratumoral STING activation with $\mathrm{T}$ cell checkpoint modulation generates systemic antitumor immunity. Cancer Immunol Res. 2017;5(8):676-684.

9. Ohkuri T, et al. STING contributes to antiglioma immunity via triggering type I IFN signals in the tumor microenvironment. Cancer Immunol Res. 2014;2(12):1199-1208

10. Foote JB, et al. A STING agonist given with OX40 receptor and PD-L1 modulators primes immunity and reduces tumor growth in tolerized mice. Cancer Immunol Res. 2017;5(6):468-479.

11. Baird JR, et al. Radiotherapy combined with novel STING-targeting oligonucleotides results in regression of established tumors. Cancer Res. 2016;76(1):50-61.

12. Ishikawa H, Barber GN. STING is an endoplasmic reticulum adaptor that facilitates innate immune signalling. Nature. 2008;455(7213):674-678.

13. De Palma M, Biziato D, Petrova TV. Microenvironmental regulation of tumour angiogenesis. Nat Rev Cancer. 2017;17(8):457-474.

14. Jain RK. Antiangiogenesis strategies revisited: from starving tumors to alleviating hypoxia. Cancer Cell. 2014;26(5):605-622.

15. Khan KA, Kerbel RS. Improving immunotherapy outcomes with anti-angiogenic treatments and vice versa. Nat Rev Clin Oncol. 2018;15(5):310-324.

16. De Palma M, Jain RK. CD4 ${ }^{+} \mathrm{T}$ cell activation and vascular normalization: two sides of the same coin? Immunity. 2017;46(5):773-775.

17. Carman CV, Martinelli R. T lymphocyte-endo- thelial interactions: emerging understanding of trafficking and antigen-specific immunity. Front Immunol. 2015;6:603.

18. Tian L, et al. Mutual regulation of tumour vessel normalization and immunostimulatory reprogramming. Nature. 2017;544(7649):250-254.

19. Allen E, et al. Combined antiangiogenic and anti-PD-L1 therapy stimulates tumor immunity through HEV formation. Sci Transl Med. 2017;9(385):eaak9679.

20. Kim C, et al. Vascular RhoJ is an effective and selective target for tumor angiogenesis and vascular disruption. Cancer Cell. 2014;25(1):102-117.

21. Park JS, et al. Normalization of tumor vessels by Tie2 activation and Ang2 inhibition enhances drug delivery and produces a favorable tumor microenvironment. Cancer Cell. 2016;30(6):953-967.

22. Fukumura D, Kloepper J, Amoozgar Z, Duda DG, Jain RK. Enhancing cancer immunotherapy using antiangiogenics: opportunities and challenges. Nat Rev Clin Oncol. 2018;15(5):325-340.

23. Demaria O, et al. STING activation of tumor endothelial cells initiates spontaneous and therapeutic antitumor immunity. Proc Natl Acad Sci USA. 2015;112(50):15408-15413.

24. Baird JR, et al. STING expression and response to treatment with STING ligands in premalignant and malignant disease. PLOS ONE 2017;12(11):e0187532.

25. Liu Y, et al. Activated STING in a vascular and pulmonary syndrome. $N$ Engl J Med. 2014;371(6):507-518.

26. Chen Q, Sun L, Chen ZJ. Regulation and function of the cGAS-STING pathway of cytosolic DNA sensing. Nat Immunol. 2016;17(10):1142-1149.

27. Jang JY, et al. VEGFR2 but not VEGFR3 governs integrity and remodeling of thyroid angiofollicular unit in normal state and during goitrogenesis. EMBO Mol Med. 2017;9(6):750-769.

28. Yang $\mathrm{H}$, et al. Soluble vascular endothelial growth factor receptor-3 suppresses lymphangiogenesis and lymphatic metastasis in bladder cancer. $\mathrm{Mol}$ Cancer. 2011;10:36.

29. Zheng H, Qian J, Carbone CJ, Leu NA, Baker DP, Fuchs SY. Vascular endothelial growth factor-induced elimination of the type 1 interferon receptor is required for efficient angiogenesis. Blood. 2011;118(14):4003-4006

30. Lin EY, et al. Progression to malignancy in the polyoma middle $\mathrm{T}$ oncoprotein mouse breast cancer model provides a reliable model for human diseases. Am J Pathol. 2003;163(5):2113-2126.

31. Chon HJ, et al. Tumor microenvironment remodeling by intratumoral oncolytic vaccinia virus enhances the efficacy of immune-checkpoint blockade. Clin Cancer Res. 2019;25(5):1612-1623. 32. Dickson PV, et al. Continuous delivery of IFN-be- ta promotes sustained maturation of intratumoral vasculature. Mol Cancer Res. 2007;5(6):531-542.

33. Jia H, Thelwell C, Dilger P, Bird C, Daniels S, Wadhwa M. Endothelial cell functions impaired by interferon in vitro: Insights into the molecular mechanism of thrombotic microangiopathy associated with interferon therapy. Thromb Res. 2018;163:105-116.

34. Sidky YA, Borden EC. Inhibition of angiogenesis by interferons: effects on tumor- and lymphocyte-induced vascular responses. Cancer Res. 1987;47(19):5155-5161.

35. Gajewski TF, Schreiber H, Fu YX. Innate and adaptive immune cells in the tumor microenvironment. Nat Immunol. 2013;14(10):1014-1022.

36. Caux C, Ramos RN, Prendergast GC, Bendriss-Vermare N, Ménétrier-Caux C. A milestone review on how macrophages affect tumor growth. Cancer Res. 2016;76(22):6439-6442.

37. Albini A, Bruno A, Noonan DM, Mortara L. Contribution to tumor angiogenesis from innate immune cells within the tumor microenvironment: implications for immunotherapy. Front Immunol. 2018;9:527.

38. Schmittnaegel M, De Palma M. Reprogramming tumor blood vessels for enhancing immunotherapy. Trends Cancer. 2017;3(12):809-812.

39. Kammertoens T, et al. Tumour ischaemia by interferon- $\gamma$ resembles physiological blood vessel regression. Nature. 2017;545(7652):98-102.

40. Ivashkiv LB, Donlin LT. Regulation of type I interferon responses. Nat Rev Immunol. 2014;14(1):36-49.

41. Terawaki S, et al. IFN- $\alpha$ directly promotes programmed cell death-1 transcription and limits the duration of T cell-mediated immunity. J Immunol. 2011;186(5):2772-2779.

42. Mandai M, Hamanishi J, Abiko K, Matsumura N, Baba T, Konishi I. Dual faces of IFN $\gamma$ in cancer progression: a role of $\mathrm{PD}-\mathrm{L} 1$ induction in the determination of pro- and antitumor immunity. Clin Cancer Res. 2016;22(10):2329-2334.

43. Sharma P, Hu-Lieskovan S, Wargo JA, Ribas A. Primary, adaptive, and acquired resistance to cancer immunotherapy. Cell. 2017;168(4):707-723.

44. Ribas A. Adaptive immune resistance: how cancer protects from immune attack. Cancer Discov. 2015;5(9):915-919.

45. Marabelle A, et al. Starting the fight in the tumor: expert recommendations for the development of human intratumoral immunotherapy (HIT-IT). Ann Oncol. 2018;29(11):2163-2174.

46. Tang C, Jiang W, Yap TA. Efficacy and toxic effects of cancer immunotherapy combinations-a double-edged sword. JAMA Oncol. 2018;4(8):1116-1117.

47. Spain L, Diem S, Larkin J. Management of toxicities of immune checkpoint inhibitors. Cancer Treat Rev. 2016;44:51-60. 